\title{
Weakly time consistent concave valuations and their dual representations
}

\author{
Berend Roorda $^{1} \cdot$ Johannes M. Schumacher ${ }^{2}$
}

Received: 19 March 2014 / Accepted: 27 May 2015 / Published online: 18 November 2015

(C) The Author(s) 2015. This article is published with open access at Springerlink.com

\begin{abstract}
We derive dual characterizations of two notions of weak time consistency for concave valuations, which are convex risk measures under a positive sign convention. Combined with a suitable risk aversion property, these notions are shown to amount to three simple rules for not necessarily minimal representations, describing precisely which features of a valuation determine its unique consistent update. A compatibility result shows that for a time-indexed sequence of valuations, it is sufficient to verify these rules only pairwise with respect to the initial valuation, or in discrete time, only stepwise. We conclude by describing classes of consistently risk averse dynamic valuations with prescribed static properties per time step. This gives rise to a new formalism for recursive valuation.
\end{abstract}

Keywords Convex risk measures · Concave valuations · Duality · Weak time consistency $\cdot$ Risk aversion

Mathematics Subject Classification (2010) 91B30 • 91G99 • 46A20 • 46N10

JEL Classification D81 · C61 · G28 · G22

Research supported in part by Netspar.

B. Roorda

b.roorda@utwente.nl

J.M. Schumacher

j.m.schumacher@tilburguniversity.edu

1 Faculty of Behavioural, Management and Social Sciences, Department of Industrial Engineering and Business Information Systems, University of Twente, P.O. Box 217, 7500 AE, Enschede, The Netherlands

2 CentER and Department of Econometrics and Operations Research, Tilburg University, P.O. Box 90153, 5000 LE, Tilburg, The Netherlands 


\section{Introduction}

Consistency of dynamic valuations, or risk measures, addresses the fundamental question how risk-adjusted valuation depends, or should depend, on degrees of information. We refer to [1] for a survey of this topic. The two main application areas are in regulation, where values correspond to capital requirements, and nonlinear pricing.

The standard notion of strong time consistency, also called dynamic consistency, or simply time consistency, postulates that two positions with identical conditional values in every state at some future date must have the same value today. This guarantees that values can be determined backward recursively. The notions of weak time consistency that we consider allow to generalize this standard recursion in a single value per state to a (finite- or infinite-dimensional) vector recursion.

The restrictiveness of standard recursion is best visible in a regulatory context. It requires that capital requirements over several periods can be determined backward recursively. Concretely, if one agrees to use, for example, Tail-Value-at-Risk at $99.5 \%$ per year, an example of a concave valuation, this would result in an overly conservative "TVaR of TVaR" outcome over two years. This indicates that a conditional requirement in a future state, no matter how well chosen, does not provide sufficient information about the conditional position in that state if it comes to determining a reasonable capital requirement today. Under weak time consistency, the accumulation of conservatism can be avoided, as shown in [12].

A common approach in nonlinear pricing is to interpret the outcomes of a concave valuation as bid prices, while ask prices derive from applying the valuation to positions with a minus sign. Often also a linear pricing operator is considered that generates intrinsic values in between bid and ask prices; see e.g. [5, 7] and Sect. 5. The point of consideration is whether a conditional position in a future state should be deemed equivalent to its conditional bid price in the sense that these two can be interchanged in a position without effect on its current bid price, as required by strong time consistency. Notice that this equivalence lets the conditional bid price also play the role of an ask price, even when they differ according to the very same conditional valuation. In order to reflect the presence of more than one type of price more fundamentally, it is a natural idea to allow a joint recursion in several prices and hence adopt weaker forms of time consistency. We refer to [13, Examples 3.8 and 3.9] for illustrations of our arguments in the context of regulation and nonlinear pricing.

We analyze two forms of weak time consistency that are still strong enough to ensure uniqueness of updates, that is, to allow at most one conditional valuation that satisfies the imposed consistency condition with respect to a given initial valuation. This means that these notions do not induce a different update than strong time consistency, but extend the set of valuations that have one.

The central notion, sequential consistency, simply requires that transitions from acceptable to unacceptable, or vice versa, should not be predictable. This is precisely the combination of the well-known concepts of (weak) acceptance and rejection consistency, (4.1a), (4.1b), which separately do not induce uniqueness of updates. Conditional consistency serves as an auxiliary, even weaker notion of time consistency. It prescribes by definition a unique update that is obtained by checking the acceptability of a position restricted to all possible events at a future date. The notions of 
sequential and conditional consistency have been introduced in [12] in a simple setting for coherent risk measures on a finite outcome space. We refer to [13] for further motivation of these concepts in a more general setting that includes nonconvex risk measures.

In this paper, we translate the main characterizations in [13] to concrete conditions for dual representations. We first show how dual representations of the unique conditionally consistent updates naturally arise as densities of measures defined in terms of initial measures. An extension of the construction addresses the case in which consistent updates fail to exist. We then give a characterization of sequential consistency, partly based on the well-known supermartingale condition for acceptance consistency [8, Proposition 4.10].

In the second part of the paper, starting with Sect. 5, we work under the assumption that a certain property holds, which was called the "supermartingale property" by Detlefsen and Scandolo [6] and which we refer to as consistent risk aversion. This assumption is quite intuitive both in a regulatory setting and in a pricing framework, and it greatly simplifies the analysis. In particular, the notions of conditional consistency and sequential consistency coincide for consistently risk averse dynamic valuations, and these properties can be characterized by three simple rules in terms of dual representations. Finally, we translate these rules to a description of the set of valuations with prescribed properties per time step and relate the extra flexibility compared to standard recursion to a joint recursion over a range of risk aversion levels.

In this paper, we consider families of valuations that are indexed concordantly with a given filtration. Time consistency will usually be discussed with respect to two given instants of time. In Sect. 6.1, we describe a compatibility property that makes it possible to apply the main results to time-indexed families of valuations without any difficulties.

\section{Setup and notation}

The setting we work in is the same as, for instance, in [8] and [9, Chap. 11], extended to incorporate continuous time. A filtered probability space $\left(\Omega, \mathcal{F},\left(\mathcal{F}_{t}\right)_{t \in \mathcal{T}}, P\right)$ is assumed to be given, with $\mathcal{T} \subset[0, T]$ a discrete or continuous time axis, $0 \in \mathcal{T}, T \in \mathcal{T}$ the finite or infinite horizon date, $\mathcal{F}_{0}=\{\emptyset, \Omega\}$, and $\mathcal{F}_{T}=\mathcal{F}$. The set $L^{\infty}:=L^{\infty}(\Omega, \mathcal{F}, P)$ is taken as the universe of all financial positions under consideration. The positions that are determinate at time $t$ are given by $L_{t}^{\infty}:=L^{\infty}\left(\Omega, \mathcal{F}_{t}, P\right)$, and $L_{t}^{0}\left(\overline{\mathbb{R}}_{+}\right)$denotes the set of all $\mathcal{F}_{t}$-measurable random variables with values in $\mathbb{R}_{+} \cup\{\infty\}$.

All inequalities, equalities and limits applied to random variables are understood in the $P$-almost sure sense. The complement of an event $F \in \mathcal{F}$ is indicated by $F^{c}$.

We define $\mathcal{Q}$ as the set of all probability measures on $(\Omega, \mathcal{F})$ that are absolutely continuous with respect to the reference measure $P$. Following [9], the symbol $\mathcal{Q}_{t}$ is used to denote the set of probability measures that are equivalent to $P$ on $\mathcal{F}_{t}$, whereas the collection of probability measures that coincide with $P$ on $\mathcal{F}_{t}$ is indicated by $\mathcal{P}_{t}$. To denote the subset of $\mathcal{Q}$ consisting of measures that are equivalent to $P$, we use the conventional symbol $\mathcal{M}^{e}(P)$ instead of $\mathcal{Q}_{T}$. 
We use the following notation related to pasting probability measures into each other, similar to the usage, for example, in [6, Definition 9]. We write $E_{t}^{Q}$ for $E^{Q}\left[\cdot \mid \mathcal{F}_{t}\right]$. For a given pair $Q^{\prime} \in \mathcal{Q}, Q \in \mathcal{Q}_{t}$, the probability measure $Q^{\prime} Q_{t} \in \mathcal{Q}$ is defined by the property

$$
E^{Q^{\prime} Q_{t}} X=E^{Q^{\prime}} E_{t}^{Q} X \quad\left(X \in L^{\infty}\right) .
$$

Note that $Q \in \mathcal{P}_{t}$ if and only if $Q=P Q_{t}$. We also make use of conditional pasting with respect to $F \in \mathcal{F}_{t}: Q^{\prime} Q_{t}^{F}$ is the probability measure in $\mathcal{Q}$ defined by

$$
E^{Q^{\prime} Q_{t}^{F}} X=E^{Q^{\prime}}\left(1_{F} E_{t}^{Q} X+1_{F^{c}} E_{t}^{Q^{\prime}} X\right) \quad\left(X \in L^{\infty}\right) .
$$

We consider conditional valuations $\phi_{t}: L^{\infty} \rightarrow L_{t}^{\infty}$ of the form

$$
\phi_{t}(\cdot)=\underset{Q \in \mathcal{Q}_{t}}{\operatorname{ess} \inf _{t}}\left(E_{t}^{Q}(\cdot)+\theta_{t}(Q)\right)
$$

where the threshold function $\theta_{t}: \mathcal{Q}_{t} \rightarrow L_{t}^{0}\left(\overline{\mathbb{R}}_{+}\right)$satisfies

$$
\underset{Q \in \mathcal{Q}_{t}}{\operatorname{essinf}} \theta_{t}(Q)=0 \text {. }
$$

As discussed in [9, Chap. 4], these are all mappings $\phi_{t}: L^{\infty} \rightarrow L_{t}^{\infty}$ with the following five properties (with $X, Y, X_{n} \in L^{\infty}, C, \Lambda \in L_{t}^{\infty}, 0 \leq \Lambda \leq 1$ ): (i) normalization: $\phi_{t}(0)=0$, (ii) monotonicity: $X \leq Y \Rightarrow \phi_{t}(X) \leq \phi_{t}(Y)$, (iii) $\mathcal{F}_{t}$-translation invariance: $\phi_{t}(X+C)=\phi_{t}(X)+C$, (iv) $\mathcal{F}_{t}$-concavity:

$$
\phi_{t}(\Lambda X+(1-\Lambda) Y) \geq \Lambda \phi_{t}(X)+(1-\Lambda) \phi_{t}(Y),
$$

and (v) continuity from above: $X_{n} \searrow X \Rightarrow \phi_{t}\left(X_{n}\right) \searrow \phi_{t}(X)$.

The class of mappings from $L^{\infty}$ to $L_{t}^{\infty}$ that satisfy these properties will be denoted by $\mathcal{C}_{t}$. We refer to its elements as concave valuations. A mapping $\phi_{t} \in \mathcal{C}_{t}$ is called coherent if it also satisfies $\mathcal{F}_{t}$-positive homogeneity, that is, $\phi_{t}(\Lambda X)=\Lambda \phi_{t}(X)$ for $X \in L^{\infty}, \Lambda \in L_{t}^{\infty}, \Lambda \geq 0$. These are precisely the elements of $\mathcal{C}_{t}$ that can be represented by a threshold function $\theta_{t}$ that only takes the values 0 and $\infty$.

Mappings with properties (i)-(iii) are called (conditional) monetary valuations or, usually with opposite sign convention, monetary risk measures. Monetary valuations possess the elementary property of

$$
\mathcal{F}_{t} \text {-regularity: } \phi_{t}\left(1_{F} X\right)=1_{F} \phi_{t}(X) \quad\left(F \in \mathcal{F}_{t}\right),
$$

which is a minimal requirement for a meaningful interpretation of the mapping $\phi_{t}$ as a normalized valuation at time $t$.

The threshold function associated to a given conditional valuation is not determined uniquely. However, to a given $\phi_{t} \in \mathcal{C}_{t}$, there is a unique minimal threshold function, which is given by

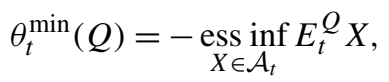


where $\mathcal{A}_{t}$ denotes the acceptance set that is defined by

$$
\mathcal{A}_{t}=\left\{X \in L^{\infty} \mid \phi_{t}(X) \geq 0\right\} .
$$

We call a threshold function $\theta_{t}$ regular if it satisfies

$$
1_{F} E_{t}^{Q}=1_{F} E_{t}^{R} \quad \Rightarrow \quad 1_{F} \theta_{t}(Q)=1_{F} \theta_{t}(R) \quad\left(Q, R \in \mathcal{Q}_{t}, F \in \mathcal{F}_{t}\right) .
$$

This property is similar to the regularity property (2.4) for risk measures. It has been called the "finite pasting property" in [8, after Lemma 3.3] and the "local property" in [11, Lemma 3.12]. Minimal threshold functions always have this property, and we sometimes impose (2.7) as a regularity condition when nonminimal thresholds are considered.

A valuation $\phi_{t} \in \mathcal{C}_{t}$ is called sensitive if

$$
X \supsetneqq 0 \quad \Rightarrow \quad \phi_{t}(X) \supsetneqq 0 \quad\left(X \in L^{\infty}\right)
$$

and strongly sensitive, or also strictly monotone, if

$$
X \supsetneqq Y \quad \Rightarrow \quad \phi_{t}(X) \supsetneqq \phi_{t}(Y) \quad\left(X, Y \in L^{\infty}\right) .
$$

\section{Conditional consistency}

Conditional consistency for a pair of valuations $\phi_{s}, \phi_{t}$ can be expressed compactly as the requirement that (cf. [13])

$$
\mathcal{A}_{t}=\mathcal{A}_{s}^{t}
$$

where $\mathcal{A}_{t}$ is the acceptance set of $\phi_{t}$, see (2.6) and $\mathcal{A}_{s}^{t}$ is the $\mathcal{F}_{t}$-restriction of $\mathcal{A}_{s}$,

$$
\mathcal{A}_{s}^{t}=\left\{X \in L^{\infty} \mid \phi_{s}\left(1_{F} X\right) \geq 0 \text { for all } F \in \mathcal{F}_{t}\right\} .
$$

A valuation $\phi_{t}$ that satisfies (3.1) for a given $\phi_{s}$ is called the conditionally consistent update of $\phi_{s}$. This update is unique because conditional monetary valuations are completely determined by their acceptance set. For coherent risk measures $\phi_{s}$, this update is simply obtained by conditioning probability measures; cf. [12, Theorem 7.1]. In other words, conditional consistency generalizes the notion of Bayesian updating. More precisely, assuming sensitivity of $\phi_{s}$ in order to avoid technicalities, the conditionally consistent update in $\mathcal{C}_{t}$ of $\phi_{s}$ is given by (cf. [13])

$$
\phi_{t}(X)=\operatorname{ess} \inf \left\{E_{t}^{Q} X \mid Q \in \mathcal{M}^{e}(P), \theta_{s}(Q)=0\right\} .
$$

As observed in [14, Remark 3.1.10], outside the coherent class, it is not guaranteed that $\mathcal{A}_{s}^{t}$ has the property

$$
1_{F} X, 1_{F^{c}} X \in \mathcal{A}_{s}^{t} \quad \Rightarrow \quad X \in \mathcal{A}_{s}^{t} \quad\left(X \in L^{\infty}\right) .
$$


Since this is a necessary condition for $\mathcal{F}_{t}$-regularity (2.4) of $\phi_{t}$ satisfying (3.1), a conditionally consistent update is not possible in $\mathcal{C}_{t}$ (and not even in the monetary class) when that property is not satisfied.

We characterize the existence of a conditionally consistent update of a given $\phi_{s}$ in terms of the operator $\eta: \mathcal{Q}_{s} \times \mathcal{F}_{t} \rightarrow L_{s}^{0}\left(\overline{\mathbb{R}}_{+}\right)$defined by

$$
\eta(Q, A)=-\operatorname{ess} \inf \left\{E_{S}^{Q}\left(1_{A} X\right) \mid 1_{A} X \in \mathcal{A}_{s}^{t}\right\} .
$$

Nonnegativity of this function follows from the fact that $0 \in \mathcal{A}_{s}^{t}$. It is also clear that $\eta$ can only take infinite values where $\theta_{S}(Q)$ is infinite because

$$
\eta(Q, A) \leq-\operatorname{ess} \inf \left\{E_{s}^{Q} X \mid X \in \mathcal{A}_{s}\right\}=\theta_{s}^{\min }(Q) \leq \theta_{s}(Q) .
$$

The function $\eta$ can be viewed as a dual representation of $\mathcal{A}_{s}^{t}$ in the sense that

$$
X \in \mathcal{A}_{s}^{t} \Longleftrightarrow E_{s}^{Q}\left(1_{A} X\right)+\eta(Q, A) \geq 0 \text { for all } A \in \mathcal{F}_{t}, Q \in \mathcal{Q}_{s} .
$$

The implication from left to right is obvious from the definition of $\mathcal{A}_{s}^{t}$, whereas the reverse implication follows from the fact that $X \notin \mathcal{A}_{s}^{t}$ implies that $E_{S}^{Q}\left(1_{A} X\right)+\theta_{s}(Q) \nsupseteq 0$ for some $Q \in \mathcal{Q}_{s}$ and $A \in \mathcal{F}_{t}$, together with inequality (3.5).

Proposition 3.1 The valuation $\phi_{s} \in \mathcal{C}_{s}$ admits a conditionally consistent update $\phi_{t}$ if and only if for all $Q \in \mathcal{Q}_{s}$, the mapping $\eta(Q, \cdot)$ is additive; in other words,

$$
\eta(Q, A \cup B)=\eta(Q, A)+\eta(Q, B) \quad \text { for all } A, B \in \mathcal{F}_{t}, A \cap B=\emptyset .
$$

If this holds, then $E^{Q} \eta(Q, \cdot)$ is a measure on $\mathcal{F}_{t}$ that is absolutely continuous with respect to $Q$, and its Radon-Nikodým derivative $\mu_{t}(Q)$ for given $Q \in \mathcal{Q}_{t}$ equals the minimal threshold $\theta_{t}^{\min }(Q)$ for the update $\phi_{t}$.

The proof is in the Appendix. We conclude this section by addressing the question how to define an update in case (3.6) does not hold and return to the main line in Sect. 4.

\subsection{The refinement update}

The $\mathcal{F}_{t}$-refinement update $\phi_{s}^{t}$ of a given sensitive $\mathcal{F}_{s}$-conditional monetary valuation $\phi_{s}$ has been introduced in [13] as the smallest $\mathcal{F}_{t}$-conditional monetary valuation whose acceptance set contains the set $\mathcal{A}_{s}^{t}$ defined in (3.2). In other words, $\phi_{s}^{t}$ is the conditional capital requirement for $\mathcal{A}_{s}^{t}[6,11]$. It is defined as [13, Definition 4.3]

$$
\phi_{s}^{t}(X)=\operatorname{ess} \sup \left\{Y \in L_{t}^{\infty} \mid \phi_{s}\left(1_{F}(X-Y)\right) \geq 0 \text { for all } F \in \mathcal{F}_{t}\right\}
$$


and its acceptance set is the closure of $\mathcal{A}_{s}^{t}$ under (3.3),

$$
\begin{array}{r}
\mathcal{B}_{s}^{t}=\left\{\sum_{i \in \mathbb{N}} 1_{A_{i}} X_{i} \mid X_{i} \in \mathcal{A}_{s}^{t}, A_{i} \in \mathcal{F}_{t}, \bigcup_{i \in \mathbb{N}} A_{i}=\Omega,\right. \\
\left.A_{i} \cap A_{j}=\emptyset \text { for } i \neq j\right\} \supseteq \mathcal{A}_{s}^{t} .
\end{array}
$$

Sensitivity of $\phi_{s}$ ensures that the mapping $\phi_{s}^{t}$ is indeed an $\mathcal{F}_{t}$-conditional monetary valuation, that is, it satisfies the first three properties of risk measures in $\mathcal{C}_{t}$ listed in Sect. 2; cf. [13, Corollary 4.3]. Concavity is preserved as well, that is, the $\mathcal{F}_{t}$-refinement update of $\phi_{s} \in \mathcal{C}_{s}$ is $\mathcal{F}_{t}$-concave; cf. [3] and [13, Proposition 2.2]. It follows from (3.1) that $\phi_{s}^{t}$ must coincide with the conditionally consistent update if that update exists. Otherwise, the inclusion in (3.8) is strict (see [13, Example 4.5] for a simple example), and we know from the previous section that this occurs when the mapping $\eta$ given by (3.4) is not additive in its second argument.

We restrict attention to $s=0$ in this section and consider a valuation $\phi_{0} \in \mathcal{C}_{0}$. Perhaps not surprisingly, the refinement update is closely related to the smallest measure $\bar{\eta}$ dominating $\eta$, which is given by

$$
\bar{\eta}(Q, A):=\sup \left\{\sum_{i \in \mathbb{N}} \eta\left(Q, A_{i}\right) \mid \bigcup_{i \in \mathbb{N}} A_{i}=A, A_{i} \cap A_{j}=\emptyset \text { for } i \neq j\right\} .
$$

Notice that $\bar{\eta}(Q)$ need not be finite even for measures $Q$ for which $\theta_{0}(Q, \cdot)$ is finite, as is illustrated by the following example.

Example 3.2 Take $\phi_{0}=\min \left\{E^{P}, E^{Q}(\cdot)+\theta\right\}$ with $\theta>0$. Assume that the space $\left(\Omega, P, \mathcal{F}_{t}\right)$ is atomless and that $Q$ is equivalent to $P$. Also assume that there exists $X \in L^{\infty}$ such that $E_{t}^{P} X=0$ and $E_{t}^{Q} X=-\theta$. Then not only $X \in \mathcal{A}_{0}^{t}$, but also $1_{A} X / Q[A] \in \mathcal{A}_{0}^{t}$ for all nontrivial $A \in \mathcal{F}_{t}$. Hence, $\eta(Q, A)=\theta$ for all such $A$, and consequently $\bar{\eta}(Q, F)=\infty$ for all nontrivial $F \in \mathcal{F}_{t}$. It follows that the refinement update is given by $E_{t}^{P}$.

We prove a representation theorem under the assumption that the update is continuous from above. The proof is in the Appendix.

Proposition 3.3 Let a sensitive concave valuation $\phi_{0} \in \mathcal{C}_{0}$ be given, and assume that the $\mathcal{F}_{t}$-refinement update $\phi_{0}^{t}$ is continuous from above. Then we have that

$$
\phi_{0}^{t}(X)=\underset{Q \in \mathcal{Q}_{t}}{\operatorname{ess} \inf _{t}}\left(E_{t}^{Q} X+\mu_{t}(Q)\right)
$$

where $\mu_{t}(Q)$ is the Radon-Nikodým derivative with respect to $Q$ of $\bar{\eta}(Q, \cdot)$ as defined in (3.9). 


\section{Characterization of sequential consistency}

We say that the conditional monetary valuations $\phi_{s}$ and $\phi_{t}$ are sequentially consistent, or that $\phi_{t}$ is a sequentially consistent update of $\phi_{s}$, if the following conditions hold (cf. [13]):

$$
\begin{aligned}
& \phi_{t}(X) \geq 0 \quad \Rightarrow \quad \phi_{S}(X) \geq 0 \quad\left(X \in L^{\infty}\right) \\
& \phi_{t}(X) \leq 0 \quad \Rightarrow \quad \phi_{s}(X) \leq 0 \quad\left(X \in L^{\infty}\right)
\end{aligned}
$$

The term "sequential" is chosen to express that the values of a given position at a sequence of time instants should not change sign predictably. These two requirements have been called weak acceptance consistency and weak rejection consistency, respectively; we use the terms "acceptance consistency" and "rejection consistency" for brevity. They can be combined into one implication that characterizes sequential consistency (cf. [13, Lemma 3.2]), namely

$$
\phi_{t}(X)=0 \quad \Rightarrow \quad \phi_{s}(X)=0 .
$$

This can be viewed as an extension of the normalization condition $\phi_{0}(0)=0$ that requires a zero outcome not only for the zero position, but also for every $X \in L^{\infty}$ such that $\phi_{t}(X)=0$. We refer to [13] for a further discussion of this concept. In particular, it is shown in this reference that sequential consistency implies conditional consistency under the assumption of strong sensitivity (2.8), so that uniqueness of updates is guaranteed. On the other hand, sequential consistency is much weaker than the standard notion of strong time consistency, which requires that

$$
\phi_{s}(X)=\phi_{s}\left(\phi_{t}(X)\right) \text {. }
$$

We further discuss this backward recursion in Sect. 7.

Acceptance consistency has been characterized by a supermartingale condition on threshold functions; see [8, Proposition 4.10]. Adapted to our setting, and with a slight generalization for risk measures that are not sensitive and thresholds that are not minimal, the result reads as follows. All proofs in this section are in the Appendix.

Lemma 4.1 Acceptance consistency (4.1a) holds for a pair $\left(\phi_{s}, \phi_{t}\right)$ with $\phi_{s} \in \mathcal{C}_{s}$ and $\phi_{t} \in \mathcal{C}_{t}$ if

$$
\theta_{s}\left(Q^{\prime} Q_{t}\right) \geq E_{s}^{Q^{\prime}} \theta_{t}(Q) \quad\left(Q^{\prime} \in \mathcal{Q}_{s}, Q \in \mathcal{Q}_{t}\right)
$$

and only if their minimal threshold functions satisfy this property.

We identify $Q \in \mathcal{Q}$ with its Radon-Nikodým derivative $z^{Q}:=\frac{d Q}{d P}$ and equip the set $\mathcal{Q}$ with the corresponding $L^{1}$-topology. The corresponding $\varepsilon$-neighborhood of $Q$ is denoted by

$$
B^{\varepsilon}(Q):=\left\{Q^{\prime} \in \mathcal{Q} \mid\left\|z^{Q^{\prime}}-z^{Q}\right\|_{1}<\varepsilon\right\}
$$


We also use the union of these sets over probability measures of the form $R Q_{t}$ for given $Q \in \mathcal{Q}_{t}$,

$$
B_{t}^{\varepsilon}(Q):=\bigcup_{R \in \mathcal{Q}} B^{\varepsilon}\left(R Q_{t}\right)
$$

Theorem 4.2 Let a pair of valuations $\phi_{s} \in \mathcal{C}_{s}, \phi_{t} \in \mathcal{C}_{t}$ be given, and suppose that these valuations are represented by regular threshold functions $\theta_{s}$ and $\theta_{t}$, respectively. The pair $\left(\phi_{s}, \phi_{t}\right)$ is sequentially consistent if we have both

$$
\begin{aligned}
\operatorname{ess} \inf \left\{\theta_{s}\left(Q Q_{t}^{*}\right)-E_{s}^{Q_{\theta_{t}}}\left(Q^{*}\right) \mid Q \in \mathcal{Q}_{s}\right\} \geq 0 & \left(Q^{*} \in \mathcal{Q}_{t}\right), \\
\inf \left\{E^{P}\left(\theta_{s}(Q)-E_{s}^{Q_{\theta_{t}}}\left(Q^{*}\right)\right) \mid Q \in B_{t}^{\varepsilon}\left(Q^{*}\right) \cap \mathcal{P}_{s}\right\} \leq 0 & \left(\varepsilon>0, Q^{*} \in \mathcal{Q}_{t}\right. \\
& \text { with } \left.\theta_{t}\left(Q^{*}\right) \text { bounded }\right),
\end{aligned}
$$

and only if these conditions hold for their minimal threshold functions.

From Theorem 4.2 we immediately obtain sufficiency of the following, simpler criterion.

Corollary 4.3 A pair of valuations $\phi_{s} \in \mathcal{C}_{s}, \phi_{t} \in \mathcal{C}_{t}$ represented by regular $\theta_{s}$ and $\theta_{t}$, respectively, is sequentially consistent if for all $Q^{*} \in \mathcal{Q}_{t}$,

$$
\operatorname{ess} \inf \left\{\theta_{s}\left(Q Q_{t}^{*}\right)-E_{s}^{Q_{\theta}}\left(Q^{*}\right) \mid Q \in \mathcal{Q}_{s}\right\}=0 .
$$

Before we discuss the interpretation, let us first briefly compare the criteria in the corollary and the preceding theorem. Criterion (4.8) is obtained by extending the requirement in (4.7b) in two respects: to $Q^{*}$ with unbounded $\theta_{t}\left(Q^{*}\right)$, and not only for $\varepsilon>0$, but also for $\varepsilon=0$. Both extensions are not without loss of generality, as shown by two counterexamples in Sect. A.6 of the Appendix.

For monetary valuations $\phi$ that are coherent, so that the minimum threshold only takes the values 0 and $\infty$, condition (4.8) amounts to the requirement that measures applied at $t$ (i.e., $Q^{*} \in \mathcal{Q}_{t}$ such that $\theta_{t}\left(Q^{*}\right)=0$ ) can be combined into one measure of the form $Q Q_{t}^{*} \in \mathcal{Q}$ with zero threshold. This property has been called junctedness in [12]. In the noncoherent case, we can again interpret (4.8) as a junctedness condition, considering $\theta_{t}\left(Q^{*}\right)$ as an affine term added to the conditional expectation of positions. The criterion requires that for all conditional affine functionals of the form $E_{t}^{Q^{*}}(\cdot)+\theta_{t}\left(Q^{*}\right)$, there exists an initial "junct" $Q \in \mathcal{Q}$ that approximately amounts to taking a weighted average of the outcome of this functional.

\section{Risk aversion in concave valuations}

In this section, we summarize some well-known properties of valuations related to risk aversion. This prepares for our definition of consistent risk aversion in the next section, which plays an important role in simplifying the characterizations obtained 
so far. We refer to [9, Chap. 2] for an extensive introduction to risk aversion, emphasizing its role in axiomatic frameworks for risk measures.

A valuation $\phi_{t} \in \mathcal{C}_{t}$ is said to exhibit risk aversion at level $t$ with respect to a measure $Q \in \mathcal{Q}$ if $\phi_{t}(X) \leq E_{t}^{Q} X$ for all $X \in L^{\infty}$. In terms of the minimal representation $\theta_{t}^{\min }$ of $\phi_{t}$, the criterion is simply

$$
\theta_{t}^{\min }(Q)=0
$$

The terminology is taken from the literature on premium principles, although the direction of the inequality is reversed here due to our different sign convention. An alternative term that is sometimes used is that there is nonnegative risk loading. In the applications to premium setting, the measure $P$ is the "physical" (real-world) measure, and the difference $E_{t}^{P}(X)-\phi_{t}(X)$ is viewed as a risk margin. A similar interpretation may be given in a regulatory context, where $\phi_{0}(X)$ serves to determine the amount of required capital associated to a risky position $X$. Alternatively, one may think of $P$ as a pricing measure and interpret $\phi_{t}(X)$ as a bid price for the payoff $X$; in other words, $\phi_{t}(X)$ is the price that a trader at time $t$ is able to get in the market for a contract that obliges the seller to deliver a contingent payoff $X$. The corresponding ask price is $-\phi_{t}(-X)[10,5,7]$; this is the price that a trader needs to pay to obtain the contingent payoff $X$. The inequality $\phi_{t}(X) \leq E_{t}^{P} X$ implies $E_{t}^{P} X \leq-\phi_{t}(-X)$, so that the "intrinsic value" $E_{t}^{P} X$ lies between the bid price $\phi_{t}(X)$ and the ask price $-\phi_{t}(-X)$. The presence of a martingale measure inducing expected values that are bracketed by the bid and ask prices associated to a nonlinear valuation $\phi_{t}$ is a wellknown condition for absence of arbitrage [10, Theorem 3.2]; cf. also the discussion in [13].

It turns out to be convenient to study the risk aversion property in combination with acceptance consistency (4.1a), which guarantees that if the most strongly aggregated valuation exhibits risk aversion, then so do its updates.

Lemma 5.1 If a concave valuation $\phi_{t} \in \mathcal{C}_{t}$ is an acceptance consistent update of a conditional valuation $\phi_{s} \in \mathcal{C}_{s}$ that exhibits risk aversion with respect to a measure $Q \in \mathcal{Q}_{t}$ at level $s$, then $\phi_{t}$ exhibits risk aversion with respect to $Q$ at level $t$.

Proof From (5.1) it follows that $\theta_{s}^{\min }(Q)=0$. The supermartingale condition (4.4) implies that the relation $\theta_{t}^{\min }(Q)=0$ holds as well, and hence $\phi_{t} \leq E_{t}^{Q}$.

In particular, if we assume that there is a measure $P^{\prime}$ equivalent to the reference measure $P$ with $\theta_{0}^{\min }\left(P^{\prime}\right)=0$, like in [15], for example, it follows that not only $\phi_{0}$, but also all its acceptance consistent updates exhibit risk aversion with respect to $P^{\prime}$. This assumption is hardly restrictive for sensitive concave valuations, as illustrated by the following lemma.

Lemma 5.2 Any coherent sensitive valuation is risk averse with respect to some $P^{\prime} \in \mathcal{M}^{e}(P)$. For a sensitive concave valuation $\phi_{0} \in \mathcal{C}_{0}$, there exists for all $\varepsilon>0$ a sensitive concave valuation $\phi_{0}^{\prime} \in \mathcal{C}_{0}$ that is risk averse with respect to some $P^{\prime} \in \mathcal{M}^{e}(P)$ and satisfies $0 \leq \phi_{0}(X)-\phi_{0}^{\prime}(X)<\varepsilon$ for all $X \in L^{\infty}$. 
Proof Let $\theta_{0}^{\min }$ denote the minimal representation of $\phi_{0} \in \mathcal{C}_{0}$. From (2.3) and (A.9) it follows that there exists $P^{\prime} \in \mathcal{M}^{e}(P)$ with $\theta_{0}^{\min }\left(P^{\prime}\right)<\varepsilon$. In the coherent case, then $\theta_{0}^{\min }\left(P^{\prime}\right)=0$. For the general case, take as $\phi_{0}^{\prime}$ the valuation obtained by redefining $\theta_{0}^{\min }\left(P^{\prime}\right):=0$. Obviously, then $0 \leq \phi_{0}(X)-\phi_{0}^{\prime}(X)<\varepsilon$ for all $X \in L^{\infty}$. Sensitivity of $\phi_{0}^{\prime}$ follows from $\phi_{0}^{\prime} \leq \phi_{0}$, and by (5.1) the claims follow.

In the sequel, we mainly concentrate on risk aversion with respect to $P$. This is without further loss of generality because formally the only role of the reference measure is to specify nullsets, and hence $P$ can be replaced by any $P^{\prime} \in \mathcal{M}^{e}(P)$.

\section{Consistent risk aversion}

The notion of risk aversion can be incorporated in time consistency in a straightforward way by imposing upper limits on $\phi_{s}(X)$ in terms of conditional expected values not only of $X$, but also of $\phi_{t}(X)$. A dynamic valuation is a family $\left(\phi_{t}\right)_{t \in \mathcal{T}}$ of conditional valuations $\phi_{t}: L^{\infty} \rightarrow L_{t}^{\infty}$.

Definition 6.1 A dynamic valuation $\left(\phi_{t}\right)_{t \in \mathcal{T}}$ is said to exhibit consistent risk aversion (CRA) with respect to $P^{\prime} \in \mathcal{M}^{e}(P)$ if $\phi_{s} \leq E_{s}^{P^{\prime}} \phi_{t}$ for all $0 \leq s \leq t \leq T$.

For $P^{\prime}=P$, we may omit the phrase "with respect to $P^{\prime \prime}$. The property of conditional risk aversion has been introduced in [6] under the name supermartingale property, and it is motivated there by the argument that the average of risk premiums at a given level of information should not exceed the risk premium that is required when less information is available. It should be noted that the sign convention in the cited paper is different from the one we use here.

Under the CRA condition, various notions of weak time consistency coincide. This is stated in the following proposition.

Proposition 6.2 Let a dynamic valuation $\phi=\left(\phi_{t}\right)_{t \in \mathcal{T}}$ be given with $\phi_{t} \in \mathcal{C}_{t}$ for all $t \in \mathcal{T}$. Under the condition that $\phi$ satisfies the CRA property with respect to some $P^{\prime} \in \mathcal{M}^{e}(P)$, the following statements are equivalent:

(i) $\phi$ is acceptance consistent;

(ii) $\phi$ is conditionally consistent;

(iii) $\phi$ is sequentially consistent.

Proof The implications from (ii) to (i) and from (iii) to (i) hold by definition, even without the CRA assumption. The CRA property directly implies rejection consistency (4.1b), so that acceptance consistency is equivalent to sequential consistency under CRA. Finally, to prove that acceptance consistency implies conditional consistency (3.1), first note that the inclusion $\mathcal{A}_{t} \subset \mathcal{A}_{s}^{t}$ already holds without the CRA assumption since acceptance consistency means that $\mathcal{A}_{t} \subset \mathcal{A}_{s}$, and by the regularity (2.4) of $\phi_{t}$, this implies that $\mathcal{A}_{t} \subset \mathcal{A}_{s}^{t}$. To derive the reverse inclusion, take $X \in \mathcal{A}_{s}^{t}$. Then $\phi_{s}\left(1_{F} X\right) \geq 0$ for all $F \in \mathcal{F}_{t}$, so that by the CRA property, $E_{s}^{P^{\prime}} 1_{F} \phi_{t}(X)=E_{s}^{P^{\prime}} \phi_{t}\left(1_{F} X\right) \geq 0$ for all $F \in \mathcal{F}_{t}$. Taking $F=\left\{\phi_{t}(X)<0\right\}$, we find that $P^{\prime}\left[\phi_{t}(X)<0\right]=0$; in other words, $\phi_{t}(X) \geq 0$, so that $X \in \mathcal{A}_{t}$. 
Recall that conditionally consistent updates are unique by definition (see (3.1)); so the combination of acceptance consistency and CRA is sufficiently strong to rule out ambiguity of updating. Hence, we can also apply the notion of CRA to an initial valuation itself as follows.

Definition 6.3 The initial valuation $\phi_{0} \in \mathcal{C}_{0}$ is said to exhibit CRA (with respect to a given filtration) if its conditionally consistent updates exist at all $t \in \mathcal{T}$ and form a CRA dynamic valuation.

We state a number of conditions that may be imposed on dynamic valuations in terms of the associated threshold functions. In Theorem 6.4, these are shown to be equivalent to consistent risk aversion of the initial valuation (with respect to $P$ ). The conditions below are stated for a given dynamic risk measure $\left(\phi_{t}\right)_{t \in \mathcal{T}}$ and for all $0 \leq s \leq t \leq T$. The proof of the theorem is in the Appendix.

Rule 1: $\theta_{t}(P)=0$.

Rule 2: $\theta_{s}\left(Q^{\prime} Q_{t}\right) \geq E_{s}^{Q^{\prime}} \theta_{t}(Q)\left(Q^{\prime} \in \mathcal{Q}_{s}, Q \in \mathcal{Q}_{t}\right)$.

Rule 3: $\theta_{s}\left(P Q_{t}\right)=E_{s}^{P} \theta_{t}(Q)\left(Q \in \mathcal{Q}_{t}\right)$.

Theorem 6.4 Let a dynamic valuation $\phi=\left(\phi_{t}\right)_{t \in \mathcal{T}}$ be given with $\phi_{t} \in \mathcal{C}_{t}$ for all $t \in \mathcal{T}$. The following four conditions are equivalent:

(1) $\phi_{0}$ exhibits consistent risk aversion, and $\phi_{t}$ is its conditionally consistent update at $t$ for all $t \in \mathcal{T}$.

(2) $\phi$ is acceptance consistent and exhibits consistent risk aversion.

(3) $\phi$ is representable by regular threshold functions $\left(\theta_{t}\right)_{t \in \mathcal{T}}$ that satisfy Rules $1-3$.

(4) The minimal threshold functions of $\phi$ satisfy Rules 1-3.

\subsection{Additional results}

Under Rule 3, the reference measure $P$ serves as a universal junct (cf. the discussion at the end of Sect. 4), guaranteeing sequential consistency in a straightforward way. Rule 3 also shows that consistent updating induces a strong link between, on the one hand, $\left.\theta_{s}\right|_{\mathcal{P}_{t}}$, that is, the threshold functions $\theta_{s}$ restricted to $\mathcal{P}_{t}$, and, on the other hand, $\theta_{t}$, whose restriction to $\mathcal{P}_{t}$ still fully describes $\phi_{t}$; cf. (A.3). It is clear that a given threshold function $\theta_{t}$ at time $t$ completely determines $\theta_{s}(Q)$ for $Q \in \mathcal{P}_{t}$. The converse is also true if we impose regularity of $\theta_{t}$.

Proposition 6.5 Let $\phi_{s}$ and $\phi_{t}$ belong to a dynamic risk measure that satisfies the first condition of Theorem 6.4. Then $\phi_{t}$ is a function of $\left.\theta_{s}^{\min }\right|_{\mathcal{P}_{t}}$ and vice versa. Moreover, for any representation $\theta_{s}$ of $\phi_{s}$, the restriction $\left.\theta_{s}\right|_{\mathcal{P}_{t}}$ determines a unique regular threshold function $\theta_{t}$ for $\phi_{t}$ that satisfies Rule 3.

Proof We show that under Rule 3, there exists at most one regular threshold function $\theta_{t}$. The second claim then follows directly, and the first claim follows from the fact that Rule 3 holds for minimal (hence regular) threshold functions. 
Given a regular threshold function $\theta_{t}$, we have $\theta_{t}\left(P Q_{t}^{A}\right)=1_{A} \theta_{t}(Q)$. (Recall that the notation $P Q_{t}^{A}$ is used for conditional pasting of the measures $P$ and $Q$; see (2.1).) Therefore, Rule 3 is equivalent to

$$
\text { Rule 3': } \theta_{s}\left(P Q_{t}^{A}\right)=E_{s}^{P} 1_{A} \theta_{t}(Q)\left(Q \in \mathcal{Q}_{t}, A \in \mathcal{F}_{t}\right) \text {. }
$$

Now consider two regular threshold functions $\theta_{t}$ and $\theta_{t}^{\prime}$ that both satisfy this rule. For given $Q \in \mathcal{Q}_{t}$, apply Rule $3^{\prime}$ to $A=\left\{\theta_{t}(Q)<\theta_{t}^{\prime}(Q)\right\}$. It can be verified directly that this event has zero probability, and by an obvious symmetry argument, it follows that $\theta_{t}(Q)$ and $\theta_{t}^{\prime}(Q)$ are equal.

The connection provided by the proposition not only reflects the uniqueness of consistent updates, which was also proved in [13] for not necessarily concave risk measures under appropriate sensitivity assumptions, but it also indicates which feature of the aggregated valuation determines the update, or why it may fail to exist. For instance, one of the consequences of Rule $3^{\prime}$ is that for disjoint $A, B \in \mathcal{F}_{t}$,

$$
\theta_{s}^{\min }\left(P Q_{t}^{A \cup B}\right)=\theta_{s}^{\min }\left(P Q_{t}^{A}\right)+\theta_{s}^{\min }\left(P Q_{t}^{B}\right)
$$

because otherwise there exists no regular time- $t$ threshold function $\theta_{t}$ that satisfies Rule $3^{\prime}$.

Remark 6.6 As shown in Proposition 6.2, the difference between conditional and sequential consistency disappears under the CRA property. In Proposition 3.1, we have characterized conditional consistency in terms of the operator $\eta$ defined in (3.4). Under the conditions of Theorem 6.4, the operator $\eta$ satisfies

$$
\eta\left(P Q_{t}, A\right)=E_{s}^{P} 1_{A} \theta_{t}^{\min }(Q)=\theta_{s}^{\min }\left(P Q_{t}^{A}\right) \quad\left(Q \in \mathcal{Q}_{t}, A \in \mathcal{F}_{t}\right) .
$$

This condition determines $\eta$ completely because $\eta\left(Q^{\prime} Q_{t}, A\right)=E_{S}^{Q^{\prime}} 1_{A} \theta_{t}^{\min }(Q)$ by Proposition 3.1. From this formula, the additivity of $\eta$, which characterizes conditional consistency, is obvious. A closer inspection reveals that if we restrict Rule $3^{\prime}$ to events $A$ that are in a sense "small," the rule still guarantees conditional consistency. To be precise, let $\mathcal{F}_{t}^{\mathrm{a}} \subset \mathcal{F}_{t}$ denote the collection of atoms of $\left(\Omega, \mathcal{F}_{t}, P\right)$, and for any given $\delta>0$, define $\mathcal{F}_{t}^{\delta}=\left\{F \in \mathcal{F}_{t} \mid P[F]<\delta\right\}$. Choose $\delta>0$ and consider the following relaxation of Rule $3^{\prime}$ :

$$
\text { Rule } 3^{\prime \prime}: \theta_{s}\left(P Q_{t}^{S}\right)=E_{s}^{P} 1_{S} \theta_{t}(Q)\left(Q \in \mathcal{Q}_{t}, S \in \mathcal{F}_{t}^{\mathrm{a}} \cup \mathcal{F}_{t}^{\delta}\right) .
$$

It reflects a weaker, "local" form of consistent risk aversion. Notice that under Rules 1,2, and $3^{\prime \prime}$, relation (6.2) still holds and that conditional consistency is preserved. It also follows that conditionally consistent updates are in fact already completely determined by the restriction of $\theta_{s}$ to $\left\{P Q_{t}^{A} \mid A \in \mathcal{F}_{t}^{\mathrm{a}} \cup \mathcal{F}_{t}^{\delta}\right\}$. Rule $3^{\prime \prime}$ is too weak, however, to guarantee that such updates are sequentially consistent since condition (6.1) may be violated for sets $A$ and $B$ such that $A \cup B$ is not in the collection $\mathcal{F}_{t}^{\mathrm{a}} \cup \mathcal{F}_{t}^{\delta}$

We conclude this section with a corollary on the characterization of CRA for initial valuations; see Definition 6.3. We make use of a compatibility result, which shows 
that it is sufficient to verify the conditions of Theorem 6.4 for a limited set of pairs of time instants $s, t$. Similar results in [13] make use of sensitivity conditions; under the CRA assumption, these conditions are not needed.

Proposition 6.7 For a dynamic valuation $\left(\phi_{t}\right)_{t \in \mathcal{T}}$ with $\phi_{t} \in \mathcal{C}_{t}$, the following statements are equivalent:

(a) The conditions of Theorem 6.4 hold for $s=0$ and for all $t \in \mathcal{T}$ with $t>0$.

(b) The conditions of Theorem 6.4 hold for all $s, t \in \mathcal{T}$ with $s<t$.

In case $\mathcal{T}=\{0,1, \ldots, T\}$ with $T$ finite, these statements are also equivalent to

(c) The conditions of Theorem 6.4 hold for all $s, t \in \mathcal{T}$ with $t=s+1$.

From Theorem 6.4 and the proof of Proposition 6.5 the following result now follows straightforwardly.

Corollary 6.8 Let an initial concave valuation $\phi_{0} \in \mathcal{C}_{0}$ be given, represented by the threshold function $\theta_{0}$. The valuation $\phi_{0}$ exhibits $C R A$ iffor all $t \in \mathcal{T}$, the (unique) regular threshold function $\theta_{t}$ that satisfies Rule $3^{\prime}$ with $s=0$ exists and satisfies Rules 1 and 2 for $s=0$. This condition is also necessary if $\theta_{0}$ is the minimal representation of $\phi_{0}$.

\section{CRA valuations with prescribed stepwise properties}

We assume a discrete, finite time axis $\mathcal{T}=\{0,1, \ldots, T\}$. Suppose that for every $t \in \mathcal{T}^{\prime}:=\{0,1, \ldots, T-1\}$, a single-period concave valuation $\bar{\psi}_{t}: L_{t+1}^{\infty} \rightarrow L_{t}^{\infty}$ is given. These single-period valuations can be composed to form a dynamic valuation $\psi=\left(\psi_{t}\right)_{t \in \mathcal{T}}$, which may be defined recursively by

$$
\psi_{T}(X)=X, \quad \psi_{t}(X)=\bar{\psi}_{t}\left(\psi_{t+1}(X)\right) \quad(t=T-1, \ldots, 0) .
$$

The dynamic valuation $\psi$ obtained in this way is strongly time consistent, that is, it satisfies (4.3). The construction as described is in fact a standard method of obtaining multiperiod strongly time consistent valuations. The given valuations $\bar{\psi}_{t}$ may correspond to one of the well-known types of static risk measures. Standard examples in the coherent class are Tail-Value-at-Risk (TVaR) and its generalization to spectral risk measures, and MINVAR and other variants of distortion measures, introduced in [4] in the context of bid-ask price modeling; see also Example 7.3. The prime example in the concave class is that of entropic risk measures, related to exponential utility.

If in general we write $\phi_{s, t}$ for the restriction $\left.\phi_{s}\right|_{L_{t}^{\infty}}$ of a concave valuation $\phi_{s}$ to $L_{t}^{\infty}$, with $t>s$, then the valuation $\psi$ defined by (7.1) satisfies $\psi_{t, t+1}=\bar{\psi}_{t}$ for all $t \in \mathcal{T}^{\prime}$, and it is in fact the only strongly time consistent dynamic valuation that has this property. However, there are in general many weakly time consistent valuations $\phi$ that satisfy the same property, that is,

$$
\phi_{t, t+1}=\bar{\psi}_{t} \quad\left(t \in \mathcal{T}^{\prime}\right) .
$$


Across a single time period, these valuations express the same level of conservatism as the given single-period valuations $\bar{\psi}_{t}$, but across multiple periods, they can avoid the piling up of conservatism that is inherent in the strongly consistent valuation $\psi$. In this section, we discuss the construction of CRA valuations that match a given set of single-period valuations. In view of Definition 6.3, the matching condition (7.2) can also be interpreted as a prescription of the stepwise properties of initial valuations $\phi_{0}$.

To simplify the analysis, we restrict attention to valuations with the additional property that for pairs $s, t \in \mathcal{T}$ with $s<t$, we have

$$
\phi_{s}(X) \leq \phi_{s}\left(E_{t}^{P} X\right)
$$

This has a natural interpretation in both a regulatory and a pricing context with $P$ respectively the real-world and a pricing measure. As shown in the next lemma, the corresponding extra rule for representations, in addition to the three for CRA, is

Rule 4: $\theta_{s}(Q) \geq \theta_{s}\left(Q P_{t}\right)\left(Q \in \mathcal{Q}_{s}\right)$.

Lemma 7.1 A valuation $\phi_{s} \in \mathcal{C}_{s}$ satisfies (7.3) for given $t>s$ if it has a representation $\theta_{s}$ satisfying Rule 4 and only if its minimal representation satisfies that rule. Furthermore, $\theta_{s, t}(Q):=\theta_{s}\left(Q P_{t}\right)$ defines a representation of $\phi_{s, t}$ if $\theta_{s}$ satisfies Rule 4.

Proof If Rule 4 holds, then

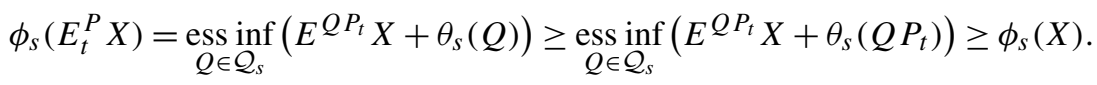

For the only if part, by (2.5) the minimal representation of $\phi_{s}$ satisfies

$$
\begin{aligned}
\theta_{s}^{\min }\left(Q P_{t}\right) & =-\underset{X \in \mathcal{A}_{s}}{\operatorname{ess} \inf _{s}} E_{\left(E_{t}^{P} X\right)} \\
& =-\operatorname{essinf}\left\{E_{s}^{Q} Z \mid Z=E_{t}^{P} X, X \in \mathcal{A}_{s}\right\} \\
& \leq-\underset{Z \in \mathcal{A}_{s}}{\operatorname{ess} \inf _{s}} E_{(Z)=\theta_{s}^{\min }(Q)}
\end{aligned}
$$

The last inequality is based on (7.3), implying that $E_{t}^{P} X \in \mathcal{A}_{s}$ if $X \in \mathcal{A}_{s}$. For the last claim, notice that because $X \in L_{t}^{\infty}$, we can always write

$$
\phi_{s, t}(X)=\operatorname{essinf}_{Q \in \mathcal{Q}_{s}, R \in \mathcal{Q}_{t}}\left(E_{s}^{Q} X+\theta_{s}\left(Q R_{t}\right)\right) .
$$

By Rule 4 we can restrict the domain to $R=P$, and the result follows.

Combining Rule 4 with the matching condition (7.2) yields a fifth rule, namely

Rule 5: $\theta_{t}\left(Q P_{t+1}\right)=\xi_{t}(Q)\left(t \in \mathcal{T}^{\prime}, Q \in \mathcal{Q}_{t}\right)$

for some regular representation $\xi_{t}$ of the to-be-matched $\bar{\psi}_{t}$ with $\xi_{t}(P)=0$. Notice that in the notation $\xi_{t}(Q)$, we identify $Q$ with its restriction to $\mathcal{F}_{t+1}$. The condition $\xi_{t}(P)=0$, which can be imposed in view of (5.1), ensures that Rule 5 for $Q=P$ is not in conflict with Rule 1. 
Corollary 7.2 A valuation $\phi_{0} \in \mathcal{C}_{0}$ exhibits $C R A$, satisfies the extra requirement (7.3) and has stepwise properties prescribed by (7.2) if and only if there exist regular threshold functions $\left(\theta_{t}\right)_{t \in \mathcal{T}}$, representing $\phi_{0}$ and its conditionally consistent updates, that satisfy Rules 1-5.

We sketch the effect of these rules backward recursively. For the last step, Rule 5 leaves no freedom, implying $\theta_{T-1}:=\xi_{T-1}$. Then, at $T-2$, the following properties of $\theta_{T-2}$ are prescribed, by resp. Rule 1,3 , and 5, for $Q \in \mathcal{Q}_{T-2}$ :

$$
\begin{aligned}
\theta_{T-2}(P) & =0 ; \\
\theta_{T-2}\left(P Q_{T-1}\right) & =E_{T-2}^{P} \theta_{T-1}(Q)=E_{T-2}^{P} \xi_{T-1}(Q) ; \\
\theta_{T-2}\left(Q P_{T-1}\right) & =\xi_{T-2}(Q) .
\end{aligned}
$$

Rules 2 and 4 both put lower bounds on $\theta_{T-2}$, which can be combined into

$$
\theta_{T-2}\left(Q^{\prime} Q_{T-1}\right) \geq \xi_{T-2}\left(Q^{\prime}\right) \vee E_{T-2}^{Q^{\prime}} \xi_{T-1}(Q) .
$$

If we impose that $\phi$ must also satisfy superrecursiveness,

$$
\phi_{s}(X) \geq \phi_{s}\left(\phi_{t}(X)\right)
$$

then this would lead to another rule, stronger than Rules 2 and 4, namely

Rule 6: $\theta_{s}\left(Q^{\prime} Q_{t+1}\right) \geq \theta_{s}\left(Q^{\prime} P_{t}\right)+E^{Q^{\prime}} \theta_{t}(Q)$,

so that the lower bound derived before would increase to

$$
\theta_{T-2}\left(Q^{\prime} Q_{T-1}\right) \geq \xi_{T-2}\left(Q^{\prime}\right)+E_{T-2}^{Q^{\prime}} \xi_{T-1}(Q) .
$$

The pattern for the remaining steps is the same, and it follows that we can take, once without and once with Rule 6,

$$
\begin{aligned}
& \theta_{t}\left(Q^{\prime} Q_{t+1}\right)=\xi_{t}\left(Q^{\prime}\right) \vee E_{t}^{Q^{\prime}} \theta_{t+1}(Q)+\hat{\theta}_{t}\left(Q^{\prime} Q_{t+1}\right), \\
& \theta_{t}\left(Q^{\prime} Q_{t+1}\right)=\xi_{t}\left(Q^{\prime}\right)+E_{t}^{Q^{\prime}} \theta_{t+1}(Q)+\hat{\theta}_{t}\left(Q^{\prime} Q_{t+1}\right),
\end{aligned}
$$

with an incremental threshold function $\hat{\theta}_{t}$ satisfying, besides regularity,

$$
\hat{\theta}_{t}\left(Q^{\prime} Q_{t+1}\right) \geq 0, \quad \text { with equality holding if } Q^{\prime} P_{t+1}=P \text { or } P Q_{t+1}=P \text {. }
$$

For $\hat{\theta}_{t}$ chosen zero in (7.6), for all $t \in \mathcal{T}^{\prime}$, the outcome is $\psi$. Choosing $\hat{\theta}_{t}$ maximal in (7.5) or (7.6), that is, infinity where zero is not prescribed, yields the maximum CRA valuation compatible with (7.2) and (7.3). This corresponds to applying risk aversion only in one period, that is, to

$$
\hat{\phi}=\left(\hat{\phi}_{t}\right)_{t \in \mathcal{T}} \quad \text { with } \quad \hat{\phi}_{t}(X)=\underset{u \in\{t, \ldots, T-1\}}{\operatorname{essinf}} E_{t}^{P} \bar{\psi}_{u}\left(E_{u+1}^{P} X\right) .
$$


Example 7.3 Let $\bar{\psi}_{t}^{\alpha}$ denote the one-step conditional valuations corresponding to $\operatorname{MINVAR}(\alpha+1)$ with parameter $\alpha \in \mathbb{R}_{+}$. For $\alpha \in \mathbb{N}$, this amounts to taking the conditional expected value of the minimum of $\alpha+1$ trials under the reference measure $P$ (cf. [4]); in particular, $\bar{\psi}_{t}^{0}(X)=E_{t}^{P}(X)$ for $X \in L_{t+1}^{\infty}$. Assume that $\alpha=n \in \mathbb{N}$ corresponds to a reasonable level of risk aversion over one period, and let $\psi^{n}$ be the recursive valuation (7.1) with stepwise valuation $\psi_{t, t+1}=\bar{\psi}_{t}^{n}$. The maximum CRA valuation satisfying (7.2) is given by (7.7), which amounts to applying $\operatorname{MINVAR}(n+1)$ in at most one period. The minimum valuation with the same stepwise properties as $\psi^{n}$ is, of course, $\psi^{n}$ itself. An example in between these extremes is obtained by setting a limit on the total number of trials until the horizon date $T,{ }^{1}$

$$
\phi_{t}^{n}(X)=\operatorname{ess} \inf \left\{\bar{\psi}_{t}^{n_{t}}\left(\cdots\left(\bar{\psi}_{T-1}^{n_{T-1}}(X)\right) \cdots\right) \mid n_{t}+\cdots+n_{T-1} \leq n\right\} .
$$

Other examples are obtained by replacing the upper bound $n$ by $n(T-t)^{\gamma}$, with $\gamma \in[0,1]$ controlling the level of risk aversion over multiple time steps.

It may be noted that for coherent valuations, as the examples just given, superrecursiveness (7.4) is equivalent to acceptance consistency (4.1a) and hence is always satisfied under CRA. Indeed, acceptance consistency is directly implied by (7.4), and conversely, (4.1a) implies that $\phi_{s}\left(X-\phi_{t}(X)\right) \geq 0$ because $\phi_{t}\left(X-\phi_{t}(X)\right)=0$, and by coherence then $\phi_{s}(X) \geq \phi_{s}\left(\phi_{t}(X)\right)+\phi_{s}\left(X-\phi_{t}(X)\right) \geq \phi_{s}\left(\phi_{t}(X)\right)$.

\subsection{Set-recursive valuation}

It may be illuminating to compare the recursive features of $\hat{\phi}$, defined by (7.7) with the standard recursive property of $\psi$ in (7.1). A backward recursive evaluation of $\hat{\phi}(X)$ for given $X \in L^{\infty}$ is quite possible if we keep track of the outcomes of a "double" value function at each time $t \in \mathcal{T}$, consisting of not only $\hat{\phi}_{t}$, but also $E_{t}^{P}$; in other words, we look at

$$
\left(\hat{\phi}_{t}(X), E_{t}^{P} X\right)=\left(\bar{\psi}_{t}\left(E_{t+1}^{P} X\right) \wedge E_{t}^{P} \hat{\phi}_{t+1}(X), E_{t}^{P} E_{t+1}^{P} X\right) .
$$

This is an example of what we call set-recursion, a generalization of the standard "singleton" recursion (4.3) in just one $\mathcal{F}_{t}$-measurable variable at $t$, as obeyed by $\psi$ in (7.1).

More generally speaking, we may consider valuations $\phi=\left(\phi_{t}\right)_{t \in \mathcal{T}}$ that are constructed by means of a recursion of the form

$$
\begin{aligned}
\Phi_{t}(X) & =\bar{\Psi}_{t}\left(\Phi_{t+1}(X)\right), \\
\phi_{t}(X) & =\bar{\phi}_{t}\left(\Phi_{t}(X)\right),
\end{aligned}
$$

where the auxiliary quantities $\Phi_{t}(X)$ take values in the sets $L_{t}^{\infty}(\Omega, \mathcal{F} ; Z)$ of essentially bounded $\mathcal{F}_{t}$-measurable functions with values in a suitable normed vector space $Z$. These auxiliary quantities are defined recursively by means of the mappings

\footnotetext{
${ }^{1}$ The given examples belong to the class of compound dynamic valuations, introduced in [13, Sect. 6], which contains an analysis of their consistency properties at a general level.
} 
$\bar{\Psi}_{t}: L_{t+1}^{\infty}(\Omega, \mathcal{F} ; Z) \rightarrow L_{t}^{\infty}(\Omega, \mathcal{F} ; Z)$, and the actual valuations at the time instants $t$ are produced from $\Phi_{t}(X)$ by applying a mapping $\bar{\phi}_{t}: L_{t}^{\infty}(\Omega, \mathcal{F} ; Z) \rightarrow L_{t}^{\infty}$. The idea is that the vector space $Z$ allows storage of multiple attributes that play a role in valuation. We now discuss this idea more concretely in terms of a parameterized family of valuations.

Definition 7.4 A parameterized family of dynamic valuations $\left(\phi^{\alpha}\right)_{\alpha \in A}$ for some in$\operatorname{dex}$ set $A$ is called set-recursive or, more specifically, A-recursive, if the following implication holds for all $X, Y \in L^{\infty}$ :

$$
\phi_{t+1}^{\alpha}(X)=\phi_{t+1}^{\alpha}(Y) \quad(\alpha \in A) \quad \Rightarrow \quad \phi_{t}^{\alpha}(X)=\phi_{t}^{\alpha}(Y) \quad(\alpha \in A) .
$$

In other words, $A$-recursiveness means that each $\phi^{\alpha}$ can be recursively specified by (7.10a), (7.10b) with $\Phi_{t}(X)=\left(\phi_{t}^{\alpha}(X)\right)_{\alpha \in A}$. We take $A \subset \mathbb{R}$, interpreted as a range of risk aversion levels. For example, if we set $\phi^{a}:=\hat{\phi}$ with $a>0$ interpreted as the overall risk aversion level of $\hat{\phi}$ defined in (7.7), and $\phi^{0}=\left(E_{t}^{P}\right)_{t \in \mathcal{T}}$, then (7.9) shows that the pair is $A$-recursive for $A=\{0, a\}$ (and $\phi^{0}$ itself for $A=\{0\}$ ).

Within the context of concave valuations, it is an obvious idea to specify $A$-recursion in terms of concave single-step valuations and to consider, for instance,

$$
\phi_{t}^{\alpha}=\underset{\alpha^{\prime} \in A}{\operatorname{ess} \inf } \bar{\psi}_{t}^{\alpha, \alpha^{\prime}} \phi_{t+1}^{\alpha^{\prime}} \text {. }
$$

Here $\bar{\psi}_{t}^{\alpha, \alpha^{\prime}}$ is a single-period valuation that specifies how conservative one can be over $[t, t+1]$, under overall risk aversion level $\alpha$, in combination with applying risk aversion level $\alpha^{\prime}$ over the remaining period. We therefore impose that $\bar{\psi}_{t}^{\alpha, \alpha^{\prime}}$ is nonincreasing in $\alpha$ and nondecreasing in $\alpha^{\prime}$. We call $\bar{\Psi}_{t}^{\alpha}:=\left(\bar{\psi}_{t}^{\alpha, \alpha^{\prime}}\right)_{\alpha^{\prime} \in A}$ the generator of $\phi^{\alpha}$ at $t$, in analogy to the standard recursive case, in which this operator is independent of $\alpha^{\prime}$ and coincides with $\phi_{t, t+1}^{\alpha} \cdot{ }^{2}$

When we take $\bar{\psi}_{t}^{\alpha, \alpha} \leq E_{t}^{P}$ (on $L_{t+1}^{\infty}$ ) for all $t \in \mathcal{T}^{\prime}$, the dynamic valuation $\phi^{\alpha}$ satisfies the CRA criterion of Definition 6.1. To obtain the equivalent conditions in Proposition 6.2, so that $\phi_{0}^{\alpha}$ is CRA (Definition 6.3), we also assume that $\bar{\psi}^{\alpha, \alpha^{\prime}}=\infty$ for $\alpha^{\prime}>\alpha$; criterion (4.1a) for acceptance consistency then follows.

This translates to dual representations as follows. Let $\theta_{t}^{\alpha, \alpha^{\prime}}$ denote a regular representation of $\bar{\psi}_{t}^{\alpha, \alpha^{\prime}}$. In order to satisfy the conditions of Theorem 6.4, we set $\theta_{t}^{\alpha, \alpha^{\prime}}(P)=0$ for all $\alpha^{\prime} \leq \alpha$. The matching condition (7.2) takes the form $\theta_{t}^{\alpha, 0}=\xi_{t}^{\alpha}$ (cf. Rule 6) with $\xi_{t}^{\alpha}$ a regular representation of the single-step valuation that has to be matched by $\phi^{\alpha}$. The corresponding representations $\theta_{t}^{\alpha}$ of $\phi_{t}^{\alpha}$ defined by (7.11) are then given by

$$
\begin{aligned}
\theta_{T-1}^{\alpha} & =\xi_{T-1}^{\alpha}, \\
\theta_{t}^{\alpha}\left(Q^{\prime} Q_{t+1}\right) & =\underset{\alpha^{\prime} \in A}{\operatorname{essinf}}\left(E_{t}^{Q^{\prime}} \theta_{t+1}^{\alpha^{\prime}}(Q)+\theta_{t}^{\alpha, \alpha^{\prime}}\left(Q^{\prime}\right)\right) .
\end{aligned}
$$

\footnotetext{
${ }^{2}$ Expression (7.11) is not without loss of generality. For instance, it can be shown that the generator of sequential TVaR, introduced in [12], takes the form (7.11) with the domain of $\alpha^{\prime}$ extended to the set $A^{\prime}$ of all $\mathcal{F}_{t+1}$-measurable variables taking values in $[0, \alpha]$, using an obvious extension of the definition of $\phi_{t+1}^{\alpha^{\prime}}$ for $\alpha^{\prime} \in A^{\prime}$.
} 
We conclude by pointing out the fact that this setting gives rise to a revision of the very definition of positions. We took as a starting point the specification of a position $X$ at some future moment $T$, and correspondingly, we can "artificially" set $\phi_{T}^{\alpha}(X)=X$ for all risk-aversion levels we consider. However, in many applications, $T$ is a somewhat arbitrarily chosen horizon date of modeling, and there is no reason to treat $T$ in a different manner than earlier time instants. So we should allow then for dependency on $\alpha$ of $\phi_{T}^{\alpha}$, and hence of a position $X$ itself, to reflect the sensitivity of $X(\omega)$ for risk aversion after $T$ for each $\omega \in \Omega$. In other words, rather than formalizing a position as $X: \Omega \rightarrow \mathbb{R}$, we could take $X^{A}: \Omega \times A \rightarrow \mathbb{R}$ as the fundamental object of valuation with $A$ a suitable range of risk aversion levels in which $X^{A}$ is monotone. It is clear that $A$-recursive valuations then become recursive in the ordinary sense and can be locally specified in terms of the newly introduced generators.

Example 7.5 The example $\phi_{0}^{n}$ in (7.8) is an $A$-recursive CRA valuation in $\mathcal{C}_{0}$ for $A=\{0, \ldots, n\}$, with generator $\bar{\Psi}_{t}^{n}=\left(\bar{\psi}_{t}^{n-k}\right)_{k \in A}$ at $t$, since we can write

$$
\phi_{t}^{n}(X)=\operatorname{ess} \inf \left\{\bar{\psi}_{t}^{n-k}\left(\phi_{t+1}^{k}(X)\right) \mid k=0, \ldots, n\right\} \leq E_{t}^{P} \phi_{t+1}^{n}(X) .
$$

To suppress the role of the horizon date $T$, we can use exponential weights $\beta^{k}$ for the parameter $n_{t+k}$ in (7.8) for some $\beta \in[0,1]$. For $T$ large and with parameters extended to $\mathbb{R}_{+}$, (7.12) then transforms into

$$
\phi_{t}^{\alpha ; \beta}(X)=\operatorname{ess} \inf \left\{\bar{\psi}_{t}^{\alpha-\beta \alpha^{\prime}}\left(\phi_{t+1}^{\alpha^{\prime}}(X)\right) \mid \alpha^{\prime} \in A\right\},
$$

with $A=[0, \alpha]$. This constitutes a recursion in "extended" conditional positions $X_{t}^{A}:=\left(\phi_{t}^{\alpha^{\prime} ; \beta}(X)\right)_{\alpha^{\prime} \in A}$, specified by the generator $\bar{\Psi}_{t}^{\alpha ; \beta}=\left(\bar{\psi}_{t}^{\alpha-\beta \alpha^{\prime}}\right)_{\alpha^{\prime} \in A}$ at $t$. Notice that the extra parameter $\beta$ in (7.13) does not affect stepwise properties and hence can be calibrated to market prices after $\alpha$ has been tuned to the market at a local time scale.

\section{Conclusions}

We have given dual characterizations of conditional and sequential consistency of concave valuations. Under the assumption of consistent risk aversion, we have characterized sequential consistency by three straightforward rules for threshold functions, and we have described the freedom still left by these rules when valuation per time step is fully prescribed. The description of set-recursive valuations in terms of generators provides a recursive structure for tuning levels of risk aversion over long and short time periods. We look upon this topic, which can be treated only to a very limited extent under strong time consistency, as an important research theme in the field of dynamic risk measures.

In particular, our analysis eventually led to a refined definition of positions, specifying their conditional value for an entire range of risk aversion levels in each state rather than for just one. For this refined specification of positions, set-recursive valuations regain the strong intuition and computational advantages of backward recursive valuation, which may facilitate the incorporation of this extra dimension in existing frameworks for nonlinear pricing and risk measurement. 
Acknowledgements We are grateful to two anonymous reviewers, the editor (Martin Schweizer), and the associate editor for their helpful comments.

Open Access This article is distributed under the terms of the Creative Commons Attribution 4.0 International License (http://creativecommons.org/licenses/by/4.0/), which permits unrestricted use, distribution, and reproduction in any medium, provided you give appropriate credit to the original author(s) and the source, provide a link to the Creative Commons license, and indicate if changes were made.

\section{Appendix}

\section{A.1 Auxiliary results}

The following lemma contains a standard result that is frequently used in the literature on dynamic risk measures. Consider two time instants $u, v \in \mathcal{T}$ with $u \leq v$. A set $\mathcal{R} \subset L_{v}^{\infty}$ is called directed downwards if for any $R, S \in \mathcal{R}$, there exists an $M \in \mathcal{R}$ with $M \leq R$ and $M \leq S$. We call $\mathcal{R} \mathcal{F}_{v}$-local if

$$
R, S \in \mathcal{R} \quad \Rightarrow \quad 1_{F} R+1_{F^{c}} S \in \mathcal{R} \quad\left(F \in \mathcal{F}_{v}\right) .
$$

Lemma A.1 If a set $\mathcal{R} \subset L_{v}^{\infty}$ is $\mathcal{F}_{v}$-local, then it is directed downwards. If a set $\mathcal{R} \subset L_{v}^{\infty}$ is directed downwards, then there exists a monotonic sequence $\left(R^{n}\right)_{n \in \mathbb{N}}$ in $\mathcal{R}$ for which $R^{n} \searrow$ ess inf $\mathcal{R}$, and

$$
E_{u}^{Q} \operatorname{ess} \inf \mathcal{R}=\operatorname{ess} \inf \left\{E_{u}^{Q} R \mid R \in \mathcal{R}\right\} \quad\left(Q \in \mathcal{Q}_{u}\right) .
$$

Proof If $\mathcal{R}$ is $\mathcal{F}_{t}$-local, then it contains with $R, S$ also $M:=1_{F} R+1_{F^{c}} S$ for $F=\{R<S\}$. Since $M \leq R$ and $M \leq S$, it follows that $\mathcal{R}$ is directed downwards. For the existence of the monotonic sequence, see, for example, [9, Theorem A.33] or [2, Remark 3.8]. The last claim follows from monotone convergence.

From this lemma we obtain the following result, which is used in Theorem 4.2. Recall that $\mathcal{P}_{t}$ denotes the subset of $\mathcal{Q}_{t}$ consisting of measures that are identical to $P$ on $\mathcal{F}_{t}$.

Lemma A.2 Let an $\mathcal{F}_{t}$-conditional monetary valuation $\phi_{t}$ be given, and assume that $\phi_{t}$ is represented by a threshold function $\theta_{t}$ that satisfies the regularity property (2.7). If $X \in L^{\infty}$ is such that $\phi_{t}(X)=0$, then for every $\varepsilon>0$, there exists a measure $Q \in \mathcal{P}_{t}$ such that

$$
E_{t}^{Q} X+\theta_{t}(Q)<\varepsilon
$$

Proof Without loss of generality, $\phi_{t} \in \mathcal{C}_{t}$ can be represented as

$$
\phi_{t}(\cdot)=\underset{Q \in \mathcal{P}_{t}}{\operatorname{ess} \inf _{t}}\left(E_{t}^{Q}(\cdot)+\theta_{t}(Q)\right)
$$

that is, with probability measures in the representation (2.2) restricted to $\mathcal{P}_{t}$; see [8, Theorem 2.3]. So with $\mathcal{R}:=\left\{E_{t}^{Q^{\prime}} X+\theta_{t}\left(Q^{\prime}\right) \mid Q^{\prime} \in \mathcal{P}_{t}\right\}, \phi_{t}(X)$ can be written as 
ess $\inf \{R \mid R \in \mathcal{R}\}$. Because $\theta_{t}$ is regular, the set $\mathcal{R}$ has the $\mathcal{F}_{t}$-local property (A.1). From Lemma A.1 it now follows that if $\phi_{t}(X)=0$, then there exists a sequence $\left(Q^{n}\right)_{n \in \mathbb{N}}$ in $\mathcal{P}_{t}$ with

$$
E_{t}^{Q^{n}} X+\theta_{t}\left(Q^{n}\right) \searrow 0 .
$$

We show that then for any $\varepsilon>0$, there exists a measure $Q \in \mathcal{P}_{t}$ that satisfies (A.2). Define $B_{n}:=\left\{E_{t}^{Q^{n}} X+\theta_{t}\left(Q^{n}\right)<\varepsilon\right\} \in \mathcal{F}_{t}, A_{0}:=B_{0}$, and $A_{n}:=B_{n} \backslash\left(\bigcup_{k=1}^{n-1} B_{k}\right)$. Due to (A.4), $\bigcup_{k=1}^{n} A_{k}=\bigcup_{k=1}^{n} B_{k} \nearrow \Omega$, so $\left(A_{n}\right)_{n \in \mathbb{N}}$ is a partition of $\Omega$. Define $Z_{n}:=d Q^{n} / d P$, and $Z:=\sum_{n \in \mathbb{N}} 1_{A_{n}} d Q^{n} / d P$. Then $Z \geq 0$ and

$$
E_{t} Z=E_{t}\left(\sum_{n \in \mathbb{N}} 1_{A_{n}} Z_{n}\right)=\sum_{n \in \mathbb{N}} 1_{A_{n}} E_{t} Z_{n}=\sum_{n \in \mathbb{N}} 1_{A_{n}}=1,
$$

where for the third equality, we used that $E_{t} Z_{n}=1$ because $Q_{n} \in \mathcal{P}_{t}$ for all $n \in \mathbb{N}$. So $Q \in \mathcal{P}_{t}$ defined by $d Q / d P=Z$ satisfies (A.2).

We remark that the claim of the lemma can be derived even more straightforwardly if regular conditional probabilities exist (cf. also [6, Definition 9]), because this allows us to choose conditional measures that satisfy the inequality (A.2) as a function of $\omega$.

\section{A.2 Proof of Proposition 3.1}

We first address the case $s=0$. We write $\mathcal{A}^{t}$ for $\mathcal{A}_{0}^{t}$.

As a first step, we prove that $\eta$ must always satisfy the subadditivity property

$$
\eta(Q, A \cup B) \leq \eta(Q, A)+\eta(Q, B) \text { for all } Q \in \mathcal{Q}, A, B \in \mathcal{F}_{t}, A \cap B=\emptyset .
$$

This follows from

$$
\begin{aligned}
-\eta(Q, A \cup B) & =\inf \left\{E^{Q}\left(1_{A} X\right)+E^{Q}\left(1_{B} X\right) \mid 1_{A \cup B} X \in \mathcal{A}^{t}\right\} \\
& \geq \inf \left\{E^{Q}\left(1_{A} X\right)+E^{Q}\left(1_{B} X\right) \mid 1_{A} X \in \mathcal{A}^{t}, 1_{B} X \in \mathcal{A}^{t}\right\} \\
& =\inf \left\{E^{Q}\left(1_{A} X\right)+E^{Q}\left(1_{B} X^{\prime}\right) \mid 1_{A} X \in \mathcal{A}^{t}, 1_{B} X^{\prime} \in \mathcal{A}^{t}\right\} \\
& =-\eta(Q, A)-\eta(Q, B) .
\end{aligned}
$$

The necessity of (3.6) is shown as follows. Assume that $\phi_{t}$ is a conditionally consistent update of $\phi_{0}$ and let its acceptance set be denoted by $\mathcal{A}_{t}$; so $\mathcal{A}_{t}=\mathcal{A}^{t}$. From (3.3), implied by the regularity property (2.4) of $\phi_{t}$, it follows that the domains in the first two lines of (A.5) coincide (in fact, also when $A$ and $B$ are not disjoint), and hence equality must hold in (A.5), so that (3.6) follows. The sufficiency of (3.6) follows from the fact that then the density of $\eta$ is well defined and represents the conditionally consistent update of $\phi_{0}$, as shown in the proof of the second claim of the proposition.

Concerning the second claim, we first prove that (3.6) implies that $\eta(Q, \cdot)$ is a measure on $\mathcal{F}_{t}$ that is absolutely continuous with respect to $Q$. For all $Q \in \mathcal{Q}, \eta(Q, \cdot)$ is nonnegative and $\eta(Q, A)=0$ for all $A$ with $Q[A]=0$; so it remains to prove 
$\sigma$-additivity. For a given $A \in \mathcal{F}_{t}$, consider a countable partition in $\mathcal{F}_{t}$ of $A$, given by $A=\bigcup_{i \in \mathbb{N}} A_{i}$ with $A_{i} \in \mathcal{F}_{t}$ for all $i$ and $A_{i} \cap A_{j}=\emptyset$ for $i \neq j$, and define

$$
\begin{aligned}
\mathcal{V}_{A} & :=\left\{1_{A} X \mid 1_{A} X \in \mathcal{A}^{t}\right\}, \\
\mathcal{W}_{A} & :=\left\{1_{A} X \mid 1_{A_{i}} X \in \mathcal{A}^{t} \text { for all } i \in \mathbb{N}\right\} .
\end{aligned}
$$

By definition, $\eta\left(Q, A_{i}\right)=-\inf \left\{E^{Q} 1_{A_{i}} X \mid 1_{A_{i}} X \in \mathcal{A}^{t}\right\}$, so for all $Q \in \mathcal{Q}$,

$$
\eta(Q, A)=-\inf \left\{E^{Q} Z \mid Z \in \mathcal{V}_{A}\right\}, \quad \sum_{i \in \mathbb{N}} \eta\left(Q, A_{i}\right)=-\inf \left\{E^{Q} Z \mid Z \in \mathcal{W}_{A}\right\} .
$$

Clearly, $\mathcal{V}_{A} \subset \mathcal{W}_{A}$, and if equality holds, then $\eta(Q, A)=\sum_{i \in \mathbb{N}} \eta\left(Q, A_{i}\right)$. We show that the assumption $\mathcal{V}_{A} \neq \mathcal{W}_{A}$ leads to a contradiction with the additivity property (3.6). If $\mathcal{V}_{A} \neq \mathcal{W}_{A}$, then there exists $X \in L^{\infty}$ such that $1_{A_{i}} X \in \mathcal{A}^{t}$ for all $i$, whereas $1_{A} X \notin \mathcal{A}^{t}$. Then $E^{Q}\left(1_{F} 1_{A} X\right)+\theta_{0}(Q)<0$ for some $Q \in \mathcal{Q}$ and $F \in \mathcal{F}_{t}$; in other words, the position determined by the restriction of $X$ to $F \cap A$ is rejected. The same must then also hold for the restriction of $X$ to $F \cap B$, where $B:=\bigcup_{i=1, \ldots, n} A_{i}$ with $n$ sufficiently large. For such $n$, it follows by (3.5) that

$$
0>E^{Q}\left(1_{B \cap F} X\right)+\theta_{0}(Q) \geq E^{Q}\left(1_{B \cap F} X\right)+\eta(Q, B) .
$$

On the other hand, because $X \in \mathcal{W}_{A}$, we have $E^{Q} 1_{A_{i} \cap F} X+\eta\left(Q, A_{i}\right) \geq 0$ for all $i$, and summation over $i=1, \ldots, n$ shows that $\eta$ is not additive. Therefore, (3.6) indeed implies that $\eta(Q, \cdot)$ is a measure on $\mathcal{F}_{t}$ that is absolutely continuous with respect to $Q$, and its Radon-Nikodým derivative $\mu_{t}(Q)$ is well defined for all $Q \in \mathcal{Q}$ up to a null set of $Q$.

To prove the last claim, still for $s=0$, we use that by the definition (3.4) of $\eta$, for any $Q \in \mathcal{Q}$,

$$
\eta(Q, A)=E^{Q} 1_{A} \mu_{t}(Q)=-\inf \left\{E^{Q} 1_{A} X \mid 1_{A} X \in \mathcal{A}^{t}\right\},
$$

and so $\inf \left\{E^{Q} 1_{A}\left(X+\mu_{t}(Q)\right) \mid 1_{A} X \in \mathcal{A}^{t}\right\}=0$. Since this holds for all $A \in \mathcal{F}_{t}$, we must have ess $\inf \left\{E_{t}^{Q}\left(X+\mu_{t}(Q)\right) \mid X \in \mathcal{A}^{t}\right\}=0$, so $\mu_{t}(Q)=-\operatorname{ess} \inf \left\{E_{t}^{Q} X \mid X \in \mathcal{A}^{t}\right\}$. From (2.5) it follows that $\mu_{t}$, restricted to $\mathcal{Q}_{t}$, is the minimum threshold function of the conditionally consistent update $\phi_{t}$ of $\phi_{0}$. This concludes the proof for the case $s=0$.

The generalization of these results to $s>0$ is straightforward from the following lemma. Here $\bar{\phi} \phi_{s}$ denotes the composition of $\bar{\phi}$ and $\phi_{s}$, that is, $\bar{\phi} \phi_{s}(X)=\bar{\phi}\left(\phi_{s}(X)\right)$.

Lemma A.3 Let concave valuations $\phi_{s} \in \mathcal{C}_{s}$ and $\phi_{t} \in \mathcal{C}_{t}$ be given, and let $\bar{\phi}$ be an unconditional valuation on $L_{s}^{\infty}$ that is normalized, monotone, and sensitive. Then the pair $\left(\phi_{s}, \phi_{t}\right)$ is conditionally consistent if and only if the pair $\left(\bar{\phi} \phi_{s}, \phi_{t}\right)$ is conditionally consistent.

Proof Let $\mathcal{A}^{\prime}$ denote the acceptance set of $\phi^{\prime}:=\bar{\phi} \phi_{s}$, and $\left(\mathcal{A}^{\prime}\right)^{t}$ its $\mathcal{F}_{t}$-restriction; see (3.2). In view of the definition (3.1) of conditional consistency, it is sufficient to prove that

$$
\left(\mathcal{A}^{\prime}\right)^{t}=\mathcal{A}_{s}^{t},
$$


that is, $\phi_{s}\left(1_{F} X\right) \geq 0$ for all $F \in \mathcal{F}_{t}$ if and only if $\bar{\phi}\left(\phi_{s}\left(1_{F} X\right)\right) \geq 0$ for all $F \in \mathcal{F}_{t}$. The forward implication follows from monotonicity and normalization of $\bar{\phi}$. For the converse implication, we prove that if $\phi_{s}\left(1_{F} X\right) \nsupseteq 0$ for some $F \in \mathcal{F}_{t}$, then $\bar{\phi}\left(\phi_{s}\left(1_{G} X\right)\right)<0$ for some $G \in \mathcal{F}_{t}$. For such $F$, consider $G:=\left\{\phi_{S}\left(1_{F} X\right)<0\right\} \in \mathcal{F}_{s}$. Then $\phi_{S}\left(1_{G} X\right)=1_{G} \phi_{s}(X) \supsetneqq 0$, and sensitivity of $\bar{\phi}$ implies that $\bar{\phi}\left(\phi_{s}\left(1_{G} X\right)\right)<0$.

By taking $\bar{\phi}$ in Lemma A.3 as a sensitive concave valuation in $\mathcal{C}_{0}$, for example, the linear operator $E^{P}$ on $L_{s}^{\infty}$, we obtain an unconditional valuation $\phi^{\prime}:=\bar{\phi} \phi_{s} \in \mathcal{C}_{0}$ for which a conditionally consistent update coincides with that of $\phi_{s}$. It remains to show that Proposition 3.1 for $\phi_{s}$ with $s>0$ is equivalent to applying it to $\phi^{\prime}$. Due to (A.6), the $\eta$-function (3.4) corresponding to $\phi^{\prime}$ can be written as

$$
\eta^{\prime}(Q, A):=-\inf \left\{E^{Q} 1_{A} X \mid 1_{A} X \in \mathcal{A}_{S}^{t}\right\} .
$$

It can be easily verified that $\eta^{\prime}(Q, \cdot)=E^{Q} \eta(Q, \cdot)$ by comparing (A.7) with (3.4) and using Lemma A.1 with $u=0, v=s$, and $\mathcal{R}$ the domain of the essential infimum in (3.4). Now the first claim of Proposition 3.1 for $s>0$ follows from the fact that $\eta(Q, \cdot)$ is additive iff $\eta^{\prime}(Q, \cdot)$ is for all $Q \in \mathcal{Q}_{s}$, and the rest follows directly.

\section{A.3 Proof of Proposition 3.3}

We write $\mathcal{A}^{t}$ for $\mathcal{A}_{0}^{t}$ as before. First, we show that for given $Q \in \mathcal{Q}, \bar{\eta}(Q, \cdot)$ is a measure that is absolutely continuous with respect to $Q$, so that $\mu_{t}$ is well defined. Nonnegativity follows from $\bar{\eta} \geq \eta \geq 0$. Furthermore, $\bar{\eta}(Q, \emptyset)=0$, and also $\bar{\eta}(Q, F)=0$ for all $F \in \mathcal{F}_{t}$ with $Q(F)=0$. It remains to show that $\bar{\eta}(Q, \cdot)$ is $\sigma$-additive. In other words, we have to show that for a given set $A \in \mathcal{F}_{t}$, with a countable partition $\left(A_{i}\right)_{i \in \mathbb{N}}$ in $\mathcal{F}_{t}$ of $A$,

$$
\bar{\eta}(Q, A)=\sum_{i \in \mathbb{N}} \bar{\eta}\left(Q, A_{i}\right) .
$$

The subadditivity of $\bar{\eta}(Q, \cdot)$ is inherited from the same property of $\eta$. That the righthand side in (A.8) is bounded from above by the left-hand side follows from (3.9) and from the fact that the countable collection of partitions $\left(A_{i}^{k}\right)_{k \in \mathbb{N}}$ of $A_{i}$ (underlying the supremum in the $i$ th term of the right-hand side) can be combined to one countable partition of $A=\bigcup_{i, k \in \mathbb{N}} A_{i}^{k}$.

Next, observe that $\phi_{0}^{t} \in \mathcal{C}_{t}$ because by assumption, $\phi_{0}^{t}$ is continuous from above, and we already mentioned after (3.7) that it satisfies the other properties that characterize $\mathcal{C}_{t}$. Define $\phi_{t}:=\phi_{0}^{t}$ with acceptance set $\mathcal{A}_{t}=\mathcal{B}_{0}^{t} \supseteq \mathcal{A}^{t}$, and let $\theta_{t}^{\text {min }}$ denote the corresponding minimal threshold function. Define the related measure $\eta^{\prime}$ by $\eta^{\prime}\left(Q^{\prime} Q_{t}, A\right):=E^{Q^{\prime}} 1_{A} \theta_{t}^{\min }(Q)$ with $Q^{\prime} \in \mathcal{Q}$ and $Q \in \mathcal{Q}_{t}$, so that we obtain $\eta^{\prime}(Q, A)=-\inf \left\{E^{Q} 1_{A} X \mid 1_{A} X \in \mathcal{A}_{t}\right\}$ for all $Q \in \mathcal{Q}$. Comparing this with the definition (3.4) of $\eta$ makes it clear that $\eta^{\prime}$ dominates $\eta$. Because $\bar{\eta}$ is the smallest measure with this property, $\bar{\eta} \leq \eta^{\prime}$, and hence $\mu_{t} \leq \theta_{t}^{\min }$. On the other hand, $\mu_{t}$ represents a valuation (call it $\tilde{\phi}_{t}$ ) with acceptance set containing $\mathcal{A}^{t}$, which can be seen as follows. For $X \in \mathcal{A}^{t}$, also $1_{A} X \in \mathcal{A}^{t}$ for all $A \in \mathcal{F}_{t}$. So by the definition of $\eta$, for all $A \in \mathcal{F}_{t}$ and $Q \in \mathcal{Q}$,

$$
E^{Q} 1_{A} X \geq-\eta(Q, A) \geq-\bar{\eta}(Q, A)=-E^{Q} 1_{A} \mu_{t}(Q),
$$


and hence $E^{Q} 1_{A}\left(E_{t}^{Q} X+\mu_{t}(Q)\right) \geq 0$, which means that $\tilde{\phi}_{t}(X) \geq 0$. Now $\tilde{\phi}_{t}$ must dominate $\phi_{t}$ because the latter is the capital requirement of $\mathcal{A}^{t}$, so that $\mu_{t} \geq \theta_{t}^{\min }$. It follows that $\theta_{t}^{\min }=\mu_{t}$ is the minimal representation of the refinement update.

\section{A.4 Proof of Lemma 4.1}

In the case in which the given valuations $\phi_{s}$ and $\phi_{t}$ are sensitive, the statement follows from [8, Proposition 4.10] combined with [8, Corollary 3.6], which states that sensitive valuations in $\mathcal{C}_{t}$ are representable by equivalent probability measures, that is,

$$
\phi_{t}(\cdot)=\operatorname{essinf}_{Q \in \mathcal{M}^{e}(P)}\left(E_{t}^{Q}(\cdot)+\theta_{t}(Q)\right) .
$$

Without the assumption that $\phi_{S}$ is sensitive, the sufficiency of (4.4) can be shown as follows. We have

$$
\begin{aligned}
\phi_{t}(X) \geq 0 \quad & \Leftrightarrow \quad E_{t}^{Q} X+\theta_{t}(Q) \geq 0 \quad\left(Q \in \mathcal{Q}_{t}\right) \\
& \Leftrightarrow \quad E_{s}^{Q^{\prime}}\left(E_{t}^{Q} X+\theta_{t}(Q)\right) \geq 0 \quad\left(Q^{\prime} \in \mathcal{Q}_{s}, Q \in \mathcal{Q}_{t}\right) \\
& \Leftrightarrow \quad E_{s}^{Q^{\prime} Q_{t}} X+E_{s}^{Q^{\prime}} \theta_{t}(Q) \geq 0 \quad\left(Q^{\prime} \in \mathcal{Q}_{s}, Q \in \mathcal{Q}_{t}\right) \\
& \Rightarrow \quad E_{s}^{Q^{\prime} Q_{t}} X+\theta_{s}\left(Q^{\prime} Q_{t}\right) \geq 0 \quad\left(Q^{\prime} \in \mathcal{Q}_{s}, Q \in \mathcal{Q}_{t}\right) \\
& \Leftrightarrow \quad \phi_{s}(X) \geq 0 .
\end{aligned}
$$

The implication in the penultimate step follows from (4.4), and for the final equivalence, we used the fact that $\mathcal{Q}_{s}=\left\{Q^{\prime} Q_{t} \mid Q^{\prime} \in \mathcal{Q}_{s}, Q \in \mathcal{Q}_{t}\right\}$ for $s, t \in \mathcal{T}$ with $s \leq t$.

Finally, the necessity of (4.4) for minimal threshold functions follows exactly as in [8, Proposition 4.10].

\section{A.5 Proof of Theorem 4.2}

The pattern of the proof is similar to that of Theorem 7.1.2 in [12], in a setting in which $\Omega$ is finite and risk measures are coherent. Throughout the largest part of the proof, we assume that $s=0$. The case $s>0$ is reduced to $s=0$ at the end of the proof.

First, we show that the criterion is sufficient, using the characterization of sequential consistency (4.2). From the first condition (4.7a), which is nothing else than a reformulation of the criterion for acceptance consistency in Lemma 4.1, it follows that

$$
\phi_{t}(X)=0 \Rightarrow \phi_{0}(X) \geq 0 \quad\left(X \in L^{\infty}\right) .
$$

The reverse inequality is implied as well, which can be seen as follows. Let $X \in L^{\infty}$ be such that $\phi_{t}(X)=0$. From Lemma A.2, which relies on the regularity of $\theta_{t}$, we obtain that for each $\varepsilon^{\prime}>0$, there exists a measure $Q^{*} \in \mathcal{P}_{t}$ such that

$$
E_{t}^{Q^{*}} X+\theta_{t}\left(Q^{*}\right)<\varepsilon^{\prime}
$$


Notice that by the definition (4.6) the density $z^{Q}$ of any $Q \in B_{t}^{\varepsilon}\left(Q^{*}\right)$ can be written as

$$
z^{Q}=z^{R Q_{t}^{*}}+\Delta \quad \text { for some } R \in \mathcal{Q}, \Delta \in L^{1} \text { with } E|\Delta|<\varepsilon,
$$

which implies that

$$
E^{Q}\left(X+\theta_{t}\left(Q^{*}\right)\right) \leq E^{R} E_{t}^{Q^{*}}\left(X+\theta_{t}\left(Q^{*}\right)\right)+b \varepsilon<\varepsilon^{\prime}+b \varepsilon
$$

with $b:=\left\|X+\theta_{t}\left(Q^{*}\right)\right\|_{\infty} \geq 0$. From (A.11) it is obvious that $b<\infty$ and also that $\theta_{t}\left(Q^{*}\right)$ is bounded. Criterion (4.7b) now implies that for all $\varepsilon>0$,

$$
\begin{aligned}
& \phi_{0}(X)=\inf \left\{E^{Q}\left(X+\theta_{t}\left(Q^{*}\right)\right)+\theta_{0}(Q)-E^{Q_{\theta_{t}}}\left(Q^{*}\right) \mid Q \in \mathcal{Q}\right\} \\
& \leq \inf \left\{E^{Q}\left(X+\theta_{t}\left(Q^{*}\right)\right)+\theta_{0}(Q)-E^{Q} \theta_{t}\left(Q^{*}\right) \mid Q \in B_{t}^{\varepsilon}\left(Q^{*}\right)\right\}
\end{aligned}
$$

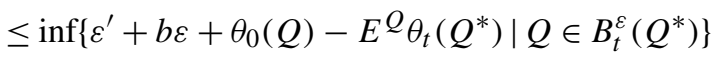

$$
\begin{aligned}
& \leq \varepsilon^{\prime}+b \varepsilon \text {. }
\end{aligned}
$$

We find that $\phi_{0}(X) \leq \varepsilon^{\prime}+b \varepsilon$ for all $\varepsilon, \varepsilon^{\prime}>0$, and hence $\phi_{0}(X) \leq 0$. Together with (A.10), this implies (4.2). This concludes the part of the proof in which we show that the criterion stated in the proposition is sufficient for sequential consistency for $s=0$.

The necessity of criterion (4.7a) for minimal thresholds is already proved by Lemma 4.1 (in fact, also for the case $s>0$, which we treat later on). It remains to prove that also (4.7b) is necessary for sequential consistency. If it did not hold, then there would exist a measure $Q^{*} \in \mathcal{Q}_{t}$ with $\theta_{t}\left(Q^{*}\right) \leq c$ for some $c \in \mathbb{R}_{+}, \varepsilon>0$, and $m>0$ such that

$$
\inf \left\{\theta_{0}(Q)-E^{Q_{\theta_{t}}}\left(Q^{*}\right) \mid Q \in B_{t}^{\varepsilon}\left(Q^{*}\right)\right\}>m .
$$

Under this condition, we derive that there exists a position $X \in L^{\infty}$ for which the implication (4.1b) does not hold. Analogously to the definitions (4.5) and (4.6), we define open sets in $L^{1}$ that contain respectively $B^{\varepsilon}\left(Q^{*}\right)$ and $B_{t}^{\varepsilon}\left(Q^{*}\right)$ by

$$
V^{\varepsilon}(Q):=\left\{z \in L^{1}|E| z-z^{Q} \mid<\varepsilon\right\}, \quad V_{t}^{\varepsilon}(Q):=\bigcup_{R \in \mathcal{Q}} V^{\varepsilon}\left(R Q_{t}\right) .
$$

Similarly to (A.12), we have $z \in V_{t}^{\varepsilon}\left(Q^{*}\right)$ if and only if

$$
z=z^{R Q_{t}^{*}}+\Delta \quad \text { for some } R \in \mathcal{Q} \text { and } \Delta \in L^{1} \text { with } E|\Delta|<\varepsilon .
$$

Define the set

$$
\mathcal{Y}:=\left\{(z, \theta) \mid z \in V_{t}^{\varepsilon}\left(Q^{*}\right), \theta \leq E z \theta_{t}\left(Q^{*}\right)+m\right\} .
$$

Because of (A.13), this is disjoint from the epigraph of $\theta_{0}$,

$$
\mathcal{Z}:=\left\{(z, \theta) \mid z=z^{Q}, Q \in \mathcal{Q}, \theta \geq \theta_{0}(Q)\right\}
$$


For an application of the separating hyperplane theorem (see e.g. [9, Theorem A.55]), we have to prove that $\mathcal{Z}$ and $\mathcal{Y}$ are convex sets and $\mathcal{Y}$ contains an interior point. The convexity of $\mathcal{Z}$ is obvious from the minimality of $\theta_{0}$. To see that $\mathcal{Y}$ is convex, we first show that $V_{t}^{\varepsilon}\left(Q^{*}\right)$ is convex. Consider $z, z^{\prime}$ in $V_{t}^{\varepsilon}\left(Q^{*}\right)$ and write $z=z^{R Q^{*}}+\Delta$, $z^{\prime}=z^{R^{\prime} Q^{*}}+\Delta^{\prime}$ for some $R, R^{\prime} \in \mathcal{Q}$ and $\Delta, \Delta^{\prime}$ as in (A.14). Then we can write $z^{\prime \prime}:=\lambda z+(1-\lambda) z^{\prime}=z^{R^{\prime \prime}} Q^{*}+\Delta^{\prime \prime}$, where we define $R^{\prime \prime}:=\lambda R+(1-\lambda) R^{\prime} \in \mathcal{Q}$ and $\Delta^{\prime \prime}=\lambda \Delta+(1-\lambda) \Delta^{\prime}$, and (A.14) therefore yields $z^{\prime \prime} \in V_{t}^{\varepsilon}\left(Q^{*}\right)$.

Next, we prove that $\mathcal{Y}$ contains an interior point of the form $\left(z^{P} Q_{t}^{*}, \theta^{\prime}\right)$. Because $\theta_{t}\left(Q^{*}\right) \leq c$, we can derive the lower bound $E z \theta_{t}\left(Q^{*}\right) \geq E \theta_{t}\left(Q^{*}\right)-c \varepsilon$ for any $z \in V^{\varepsilon}\left(P Q_{t}^{*}\right)$. Now take $\theta^{\prime}=E \theta_{t}\left(Q^{*}\right)+m-2 c \varepsilon$, so that $\theta^{\prime}+c \varepsilon \leq E z \theta_{t}\left(Q^{*}\right)+m$ for all $z \in V^{\varepsilon}\left(P Q_{t}^{*}\right)$. Then $\left(z^{P Q_{t}^{*}}, \theta^{\prime}\right)$ is an interior point because $\left(z, \theta^{\prime}+\delta\right) \in \mathcal{Y}$ for all $z \in V^{\varepsilon}\left(P Q_{t}^{*}\right)$ and $-c \varepsilon<\delta<c \varepsilon$.

Now the separating hyperplane theorem guarantees the existence of a nonzero continuous linear functional $\ell$ on $L^{1} \times \mathbb{R}$ such that $\ell(y) \geq \ell(z)$ for all $y \in \mathcal{Y}, z \in \mathcal{Z}$. Consequently, there exist $X \in L^{\infty}$ and $k \in \mathbb{R}$, not both zero, such that

$$
\begin{array}{ll}
E^{Q} X+k \theta \geq 0 & \left(\left(z^{Q}, \theta\right) \in \mathcal{Z}\right), \\
E^{Q} X+k \theta \leq 0 & \left(\left(z^{Q}, \theta\right) \in \mathcal{Y}\right) .
\end{array}
$$

First, we address the case $k \neq 0$. Inequality (A.16) implies that for any $Q \in \mathcal{Q}$, $E^{Q Q_{t}^{*}} X+k \theta \leq 0$ for all $\theta \leq E^{Q_{\theta_{t}}}\left(Q^{*}\right)+m$, in particular for all $\theta<0$, hence $k>0$. By replacing $X$ by $X / k$, we can rescale to $k=1$. Then (A.15) implies that $\phi_{0}(X) \geq 0$. On the other hand, from (A.16) it follows that for all $Q \in B^{\varepsilon}\left(Q Q_{t}^{*}\right)$,

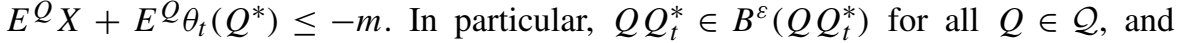
hence $E^{Q} E_{t}^{Q^{*}}\left(X+\theta_{t}\left(Q^{*}\right)\right) \leq-m$ for all $Q \in \mathcal{Q}$. This can only be true if $E_{t}^{Q^{*}}\left(X+\theta_{t}\left(Q^{*}\right)\right) \leq-m$. Therefore, $\phi_{t}(X) \leq-m$, whereas $\phi_{0}(X) \geq 0$, so that rejection consistency (4.1b) is violated.

In the case $k=0, X$ is nonzero. Inequality (A.15) implies that $E^{Q} X \geq 0$ for all $Q \in \mathcal{Q}$ with $\theta_{0}(Q)<\infty$, so $\phi_{0}(\lambda X) \geq 0$ for all $\lambda \geq 0$. Inequality (A.16) for $k=0$ implies that for all $Q \in \mathcal{Q}, E^{Q Q_{t}^{*}} X+E \Delta X \leq 0$ for all $\Delta \in L^{1}$ with $E|\Delta|<\varepsilon$ (cf. (A.14)). Because $X \neq 0$, there exists such a $\Delta$ with $E \Delta X>\varepsilon^{\prime}>0$. So $E^{Q} Q_{t}^{*} X<-\varepsilon^{\prime}$ for all $Q \in \mathcal{Q}$, and hence $E_{t}^{Q^{*}} X<-\varepsilon^{\prime}$. Take $\lambda=(c+1) / \varepsilon^{\prime}$; then $\phi_{t}(\lambda X) \leq E_{t}^{Q^{*}} \lambda X+\theta_{t}\left(Q^{*}\right) \leq-1$. So also in the case $k=0$, rejection consistency (4.1b) is violated.

The generalization to $s>0$ is straightforward on the basis of the following lemma, which is analogous to Lemma A.3.

Lemma A.4 Let $\phi_{s} \in \mathcal{C}_{s}$ and $\phi_{t} \in \mathcal{C}_{t}$ be given, and let $\bar{\phi}$ be a strongly sensitive, normalized, and monotone valuation on $L_{s}^{\infty}$. The following conditions are equivalent:

(1) $\left(\phi_{s}, \phi_{t}\right)$ is sequentially consistent.

(2) $\left(\bar{\phi} \phi_{s}, \phi_{t}\right)$ is sequentially consistent.

(3) $\left(E^{S} \phi_{s}, \phi_{t}\right)$ is sequentially consistent for all $S \in \mathcal{Q}$.

Proof To see that the first condition implies the second, assume that $\left(\phi_{s}, \phi_{t}\right)$ is sequentially consistent, and consider $X$ such that $\phi_{t}(X)=0$. From (4.2) then 
$\phi_{S}(X)=0$, and hence also $\bar{\phi}\left(\phi_{S}(X)\right)=0$; so by the same criterion (4.2) the second condition follows. Analogously, the third condition follows from $E^{S}\left(\phi_{S}(X)\right)=0$ for all $S \in \mathcal{Q}$.

Conversely, assume that $\left(\bar{\phi} \phi_{s}, \phi_{t}\right)$ is sequentially consistent and again consider $X$ such that $\phi_{t}(X)=0$. Then also $\phi_{t}\left(1_{F} X\right)=0$ for all $F \in \mathcal{F}_{t}$, in particular for all $G \in \mathcal{F}_{s}$, so by (4.2) $\bar{\phi}\left(\phi_{s}\left(1_{G} X\right)\right)=\bar{\phi}\left(1_{G} \phi_{s}(X)\right)=0$ for all $G \in \mathcal{F}_{s}$. Because $\bar{\phi}$ is strongly sensitive, it follows that $\phi_{s}(X)=0$, and hence $\left(\phi_{s}, \phi_{t}\right)$ must be sequentially consistent. So the second condition implies the first one. Applying the same argument with $\bar{\phi}:=E^{S}$ and $S \in \mathcal{Q}_{s}$ shows that also the third condition implies the first one.

Lemma A.4 implies that $\left(\phi_{s}, \phi_{t}\right)$ is sequentially consistent iff (4.7a) and (4.7b) hold for all pairs $\left(E^{S} \theta_{s}, \theta_{t}\right)$ in which $S$ belongs to $\mathcal{Q}_{s}$ and both $\theta_{s}$ and $\theta_{t}$ are minimal, because $E^{S} \theta_{s}$ is then the minimum threshold function of $E^{S} \phi_{s}$. This yields

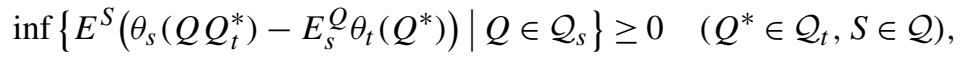

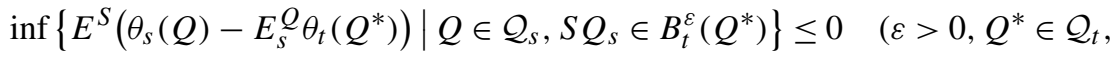

$$
\begin{aligned}
& \theta_{t}\left(Q^{*}\right) \text { bounded, } \\
& S \in \mathcal{Q}) \text {. }
\end{aligned}
$$

The first line above is equivalent to (4.7a). From Lemma A.4 with $\phi$ taken as $E^{P}$ on $L_{s}^{\infty}$ it follows that we can restrict to $S=P$ (in one or both inequalities), and for the second line, this yields (4.7b).

\section{A.6 Two counterexamples related to Corollary 4.3}

We first give an example of a sequentially consistent pair $\left(\phi_{0}, \phi_{1}\right)$ that does not satisfy (4.7b) extended to unbounded $\theta_{1}\left(Q^{*}\right)$. Let $\Omega$ consist of pairs $(v, j)$ with $v \in \mathbb{N}, j \in\{u, d\}$, and let the reference measure $P$ be defined by $P[v]=2^{-v}$, $P[u \mid v]=P[d \mid v]=\frac{1}{2}$. Take $\phi_{1}(X)(v)=\min \left\{E^{P}(X \mid v), E^{Q^{*}}(X \mid v)+v\right\}$ with $Q^{*}$ defined by $Q^{*}[v]=P[v]$ (so $Q^{*} \in \mathcal{P}_{1}$ ) and $Q^{*}[u \mid v]=\frac{1}{4}$; we write $E^{P}(X \mid v)$ for $\left(E_{1}^{P} X\right)(v)$, and so on. The minimal representation $\theta_{1}$ of $\phi_{1}$ then has $\theta_{1}(P)=0$ and $\theta_{1}\left(Q^{*}\right)(v)=v$. Take $\phi_{0}(X)=\inf E_{1}^{P} X$, which has minimal representation $\theta_{0}$ given by $\theta_{0}(Q)=0$ for $Q \in \mathcal{S}$ and $\theta_{0}(Q)=\infty$ for $Q \notin \mathcal{S}$, with $\mathcal{S}=\left\{S P_{1} \mid S \in \mathcal{Q}\right\}$.

Sequential consistency is derived from criterion (4.2) as follows. Assume that $\phi_{1}(X)=0$. Then for all $v \in \mathbb{N}$, (i) $E^{P}(X \mid v)=0$ or (ii) $E_{1}^{Q^{*}}(X \mid v)=-v$. Because (ii) cannot hold for $v>\|X\|_{\infty}$, (i) holds for such $v$, and hence $\phi_{0}(X)=0$. So (4.2) holds.

However, the inequality in (4.7b) is not satisfied for $Q^{*}$. This follows from the fact that $\mathcal{S}$ defined before is disjoint from $B_{1}^{\varepsilon}\left(Q^{*}\right)$ for $\varepsilon$ sufficiently small; in fact, this holds already for $\varepsilon=\frac{1}{2}$ because for $S, R \in \mathcal{Q}, \Delta:=z^{S P_{1}}-z^{R Q_{1}^{*}}$ is given by $\Delta(v, u)=\left(S(v)-\frac{1}{2} R(v)\right) 2^{v}$ and $\Delta(v, d)=\left(S(v)-\frac{3}{2} R(v)\right) 2^{v}$, so that we obtain $E|\Delta| \geq E 1_{u} \Delta-E 1_{d} \Delta=\frac{1}{4}+\frac{1}{4}=\frac{1}{2}$.

The second example shows that also when all threshold functions are bounded, criterion (4.8) is not necessary, that is, criterion (4.7b) cannot be imposed for $\varepsilon=0$ without loss of generality. 
In the same setting as the previous example, now consider $\phi_{0}$ given by

$$
\phi_{0}(X)=\min \left\{\inf E_{1}^{P} X, E^{Q^{*}} X+h\right\}
$$

with $h=1$ and $Q^{*}$ given by $Q^{*}[v]=2^{-v}$ and $Q^{*}[u \mid v]=1 / 2(1-f(v))$ with $f(v):=2^{-v}$ (crucial is that $f(v) \rightarrow 0$ and $f(v)>0$ for all $v$ ). That the threshold $h$ is minimal follows from $E^{Q^{*}} X=-1$ for $X \in \mathcal{A}$ given by $X(1)=(2,-2)$ and $X(v)=0$ for $v>1$; cf. (2.5). For $\phi_{1}$, we take $\phi_{1}(X)=E_{1}^{P} X \wedge\left(E_{1}^{Q^{*}} X+f\right)$, so that $\bar{f}:=E^{Q^{*}} f=\sum_{v=1}^{\infty} 2^{-2 v}=1 / 3$ (crucial is that $E^{Q^{*}} f=\bar{f}<h$ and $\lim f(v)=0$ ).

Notice that (4.8) is not satisfied for $Q^{*}$ since the domain of the infimum is just the singleton $Q^{*}$ and the outcome is $h-\bar{f}=2 / 3>0$. Nevertheless, the pair is sequentially consistent (and hence satisfies the criterion of Theorem 4.2), which we prove by deriving (4.2). Consider $X$ with $\phi_{1}(X)=0$. It is clear that $\phi_{0}(X) \geq 0$. So for all $v,\left(E_{1}^{P} X\right)(v)=0$ or $\left(E_{1}^{Q^{*}} X\right)(v)=-f(v)$. If there is a $v$ with $\left(E_{1}^{P} X\right)(v)=0$, then also $\phi_{0}(X)=0$. But also if $E_{1}^{P} X>0$, then it follows from $E_{1}^{Q^{*}} X=-f$ that $\lim _{v}\left(E_{1}^{P} X\right)(v)=0$, and also in this case $\phi_{0}(X)=0$ is implied. This proves that the pair is sequentially consistent.

\section{A.7 Proof of Theorem 6.4}

To prepare for the proof, define $\psi_{s} \in \mathcal{C}_{s}$ by $\psi_{s}:=E_{s}^{P} \phi_{t}$. Applying Lemma A.1 in a similar way as in the proof of Lemma A.2 yields that

$$
\begin{aligned}
\psi_{s}(X) & =E_{s}^{P}\left(\operatorname{ess} \inf \left\{E_{t}^{Q} X+\theta_{t}(Q) \mid Q \in \mathcal{Q}_{t}\right\}\right) \\
& =\operatorname{ess} \inf \left\{E_{s}^{P Q_{t}} X+E_{s}^{P} \theta_{t}(Q) \mid Q \in \mathcal{Q}_{t}\right\} .
\end{aligned}
$$

So $\psi_{s}$ is represented by $\theta_{s}^{\psi}$ defined by

$$
\theta_{s}^{\psi}(Q)=E_{s}^{P} \theta_{t}(Q) \quad \text { for } Q \in \mathcal{P}_{t}, \quad \theta_{s}^{\psi}(Q)=\infty \quad \text { for } Q \notin \mathcal{P}_{t} .
$$

The equivalence $1 \Leftrightarrow 2$ follows directly from Definition 6.3 and Proposition 6.2.

The implication $3 \Rightarrow 2$ is derived as follows. According to Lemma 4.1, Rule 2 implies acceptance consistency. Rule 3 in combination with (A.17) implies that

$$
\psi_{s}(X)=\operatorname{ess} \inf \left\{E_{s}^{P Q_{t}} X+\theta_{s}\left(P Q_{t}\right) \mid Q \in \mathcal{Q}_{t}\right\} .
$$

It follows that $\psi_{s} \geq \phi_{s}$, which is precisely the CRA property for $\phi$.

Next, we prove the implication $2 \Rightarrow 4$. It follows directly from Lemma 4.1 that the collection of threshold functions $\left(\theta_{t}\right)_{t \in \mathcal{T}}$ satisfies Rule 2. CRA, that is, the requirement $\phi_{s} \leq \psi_{s}$, implies that $\theta_{s}^{\min } \leq \theta_{s}^{\psi}$, so that (A.18) induces Rule 3. We already saw that CRA also implies Rule 1 . The proof is completed by noting that the implication $4 \Rightarrow 3$ is trivial.

\section{A.8 Proof of Proposition 6.7}

It is clear that (b) implies (a) and, in case $\mathcal{T}$ is finite and discrete, also (c). That (c) in that case implies (a) follows from the law of iterated expectations. It remains to prove 
that (a) implies (b). Under (a), there exist regular threshold functions $\theta_{t}$ of $\phi_{t}$ for all $t \in \mathcal{T}$ (e.g., their minimal ones) that satisfy Rules 1-3 for all pairs $s, t \in \mathcal{T}$ with $0=s<t$. We prove that the same rules must then apply to these threshold functions for all $0<s<t$.

For a given measure $Q \in \mathcal{Q}_{t}$, define $G \in \mathcal{F}_{s}$ by $G:=\left\{\theta_{s}\left(P Q_{t}\right)<E_{s}^{P} \theta_{t}(Q)\right\}$. Suppose that $P[G]>0$. Because $\theta_{t}$ is regular, we then have $\theta_{s}\left(P Q_{t}^{G}\right) \supsetneqq E_{s}^{P} 1_{G} \theta_{t}(Q)$. This implies that $E^{P} \theta_{s}\left(P Q_{t}^{G}\right)<E^{P} \theta_{t}\left(P Q_{t}^{G}\right)$, whereas Rule 3 with $s=0$ requires that both sides are equal to $\theta_{0}\left(P Q_{t}\right)$. Consequently, we must have $P[G]=0$. Analogous reasoning applies when the inequality in the definition of $G$ is reversed. It follows that Rule 3 holds for $s>0$.

In a similar way, we prove that Rule 2 holds for $s>0$. For given measures $Q^{\prime} \in \mathcal{Q}_{s}$ and $Q \in \mathcal{Q}_{t}$, define $G \in \mathcal{F}_{s}$ by $G:=\left\{\theta_{s}\left(Q^{\prime} Q_{t}\right)<E_{s}^{Q^{\prime}} \theta_{t}(Q)\right\}$. Again, suppose that $P[G]>0$. We then have $\theta_{s}(R) \supsetneqq E_{s}^{R} \theta_{t}(R)$ where $R$ is defined by $R=R^{\prime} P_{t}^{G^{c}}$ with $R^{\prime}:=Q^{\prime} Q_{t}$. Taking unconditional expected values under $P$ on both sides of the inequality and using Rule 3 with $s=0$, we obtain that $\theta_{0}\left(P R_{S}\right) \supsetneqq E^{P R_{s}} \theta_{t}(R)$. This violates Rule 2 for $s=0$. It follows that $P[G]=0$, which means that Rule 2 holds for $s>0$.

\section{References}

1. Acciaio, B., Penner, I.: Dynamic risk measures. In: Di Nunno, G., Øksendal, B. (eds.) Advanced Mathematical Methods for Finance, pp. 1-34. Springer, Heidelberg (2011)

2. Cheridito, P., Delbaen, F., Kupper, M.: Coherent and convex monetary risk measures for unbounded càdlàg processes. Finance Stoch. 9, 369-387 (2005)

3. Cheridito, P., Delbaen, F., Kupper, M.: Dynamic monetary risk measures for bounded discrete-time processes. Electron. J. Probab. 11, 57-106 (2006)

4. Cherny, A., Madan, D.B.: New measures for performance evaluation. Rev. Financ. Stud. 22, 25712606 (2009)

5. Cherny, A., Madan, D.B.: Markets as a counterparty: an introduction to conic finance. Int. J. Theor. Appl. Finance 13, 1149-1177 (2010)

6. Detlefsen, K., Scandolo, G.: Conditional and dynamic convex risk measures. Finance Stoch. 9, 539$561(2005)$

7. Eberlein, E., Madan, D., Pistorius, M., Schoutens, W., Yor, M.: Two price economies in continuous time. Ann. Finance 10, 71-100 (2014)

8. Föllmer, H., Penner, I.: Convex risk measures and the dynamics of their penalty functions. Stat. Decis. 24, 61-96 (2006)

9. Föllmer, H., Schied, A.: Stochastic Finance. An Introduction in Discrete Time, 3rd edn. Walter de Gruyter, Berlin (2011)

10. Jouini, E., Kallal, H.: Martingales and arbitrage in securities markets with transaction costs. J. Econ. Theory 66, 178-197 (1995)

11. Klöppel, S., Schweizer, M.: Dynamic indifference valuation via convex risk measures. Math. Finance 17, 599-627 (2007)

12. Roorda, B., Schumacher, J.M.: Time consistency conditions for acceptability measures, with an application to Tail Value at Risk. Insur. Math. Econ. 40, 209-230 (2007)

13. Roorda, B., Schumacher, J.M.: Membership conditions for consistent families of monetary valuations. Stat. Risk. Model. 30, 255-280 (2013)

14. Tutsch, S.: Konsistente und konsequente dynamische Risikomaße und das Problem der Aktualisierung. PhD thesis, Humboldt-Universität, Berlin (2006). Available online at http://edoc.hu-berlin. de/dissertationen/tutsch-sina-2006-11-06/PDF/tutsch.pdf

15. Tutsch, S.: Update rules for convex risk measures. Quant. Finance 8, 833-843 (2008) 